\title{
Electrochemical state and internal variables estimation using a reduced-order physics-based model of a lithium-ion cell and an extended Kalman filter
}

\author{
Kirk D. Stetzel ${ }^{\mathrm{a}}$, Lukas L. Aldrich ${ }^{\mathrm{a}}$, M. Scott Trimboli ${ }^{\mathrm{a}}$, Gregory L. Plett ${ }^{\mathrm{a}, 1, *}$ \\ ${ }^{a}$ Department of Electrical and Computer Engineering, University of Colorado Colorado Springs, Colorado Springs, CO 80918, United States
}

\begin{abstract}
This paper addresses the problem of estimating the present value of electrochemical internal variables in a lithium-ion cell in real time, using readily available measurements of cell voltage, current, and temperature. The variables that can be estimated include any desired set of reaction flux and solid and electrolyte potentials and concentrations at any set of one-dimensional spatial locations, in addition to more standard quantities such as state of charge. The method uses an extended Kalman filter along with a one-dimensional physics-based reduced-order model of cell dynamics. Simulations show excellent and robust predictions having dependable error bounds for most internal variables.
\end{abstract}

Keywords: Lithium ion battery, electrochemical model, battery management systems, state of charge estimation, electrochemical state estimation, extended Kalman filter

\section{Introduction}

This paper addresses the problem of estimating the present value of electrochemical internal variables in a lithiumion cell in real time, using readily available measurements of cell voltage, current, and temperature. The variables that can be estimated include any desired set of reaction flux and solid and electrolyte potentials and lithium concentrations at any set of one-dimensional spatial locations, in addition to more standard quantities such as electrode or cell state of charge (SOC). The method uses an extended Kalman filter (EKF) along with a one-dimensional physics-based reduced-order model (ROM) of cell dynamics.

This work is motivated by the needs of next-generation battery-management systems (BMS). A BMS needs accurate cell state estimates to be able to manage the battery pack properly to ensure safe, robust, and reliable operation. Whereas state-of-the-art battery-control strategies address short-term objectives using voltage-based design limits, little has been done to improve battery performance and to extend lifetime using design limits based on internal electrochemical variables. Just as a present-generation BMS requires state-of-charge estimates to enforce voltage limits, next-generation BMS will require estimates of internal electrochemical variables to enforce the more advanced design limits.

\footnotetext{
${ }^{*}$ Corresponding author

Email addresses: kstetzel@uccs .edu (Kirk D. Stetzel), laldrich@uccs . edu (Lukas L. Aldrich), mtrimbol@uccs . edu (M. Scott Trimboli), gplett@uccs . edu (Gregory L. Plett)

${ }^{1}$ Tel: +1 719255 3468; fax: +1 7192553589
} 
It is quite common now to see articles proposing methodologies to estimate cell state of charge. Various approaches exist [1], but model-based methods seem to be dominant as the built-in feedback makes them robust, and a good model makes them accurate.

In the estimation literature as a whole, the Kalman filter (KF) remains one of the most widely used model-based state estimators due to its simplicity, optimality, tractability and robustness [2]. Relying on an underlying linear model of system dynamics, the KF uses input-output measurements and probabilistic descriptions of uncertainty to generate a minimum-mean-squared optimal estimate of a system's internal state vector as it evolves in time. It is well known, however, that application of the KF to nonlinear systems can be difficult. A popular approach to overcome this difficulty is the implementation of an extended Kalman filter (EKF), which essentially linearizes the nonlinear system models in such a way that the linear KF steps can be used.

Model-based methods such as the KF and EKF require a mathematical description - that is, a model-of cell dynamics as part of the procedure for estimating internal states of the cell. In the electrochemical literature, these models tend to fall into one of two categories: those developed empirically by observing input-output behaviors and proposing equations to match those observations, and those developed from physics first principles.

Empirical models of cells are most commonly realized as equivalent-circuit models, since interconnected linear (and sometimes nonlinear) electric circuit elements are employed as analogs to observed behaviors [3]. These kinds of models are very commonly used in present-generation BMS implementations as they are relatively simple to develop and can predict cell input-output behaviors well so long as great care is taken in the selection of the model parameter values. Prior work shows good results for SOC-estimation using EKF with equivalent-circuit models [4-6], and indicates that model-based estimation using equivalent-circuit models may be adequate if the BMS requires estimates of SOC only and if cell aging can be captured sufficiently via slow adaptation of cell resistance and capacity parameter values.

Equivalent-circuit models can produce accurate predictions of cell voltage; however, they do not provide insight into the internal electrochemical variables of the cell. On the other hand, physics-based porous-electrode models do have this ability. Doyle, Fuller, and Newman [7, 8] have developed such a physics-based model, which comprises coupled nonlinear partial-differential equations (PDEs). While physics-based models are far more challenging to derive, they offer two significant advantages over equivalent-circuit models: first, they predict cell operation outside the bounds of previously collected data (i.e., they extrapolate well) and, second, they describe the electrochemical internal dynamics of the cell in addition to being able to predict cell voltage.

This latter feature is of critical importance to next-generation BMS. The cell's internal dynamics are key to how it ages and degrades; thus, being able to estimate the present value of these internal electrochemical variables using a physics-based model is key to predicting and invoking controls to avoid degradation.

Control algorithms based on knowledge of the internal electrochemical state have the potential to expand the performance and extend the life of cells. They can predict power limits with respect to electrode surface depletion/saturation conditions and with respect to side reactions responsible for damage and sudden loss of power [9, 10]. 
For example, some early "full-information" controls (i.e., which assume perfect knowledge of cell internal variables, as precursor work to practical implementable methods which must estimate the values of these variables using only measurements of voltage, current, and temperature) [11] shows that electrochemically limited pulse charging a 6 Ah cell to the same negative-electrode phase-potential $\phi_{s}-\phi_{e}$ at the negative-electrode/separator boundary as encountered at equilibrium at $100 \%$ SOC increases usable charge power by $22 \%$ and usable energy by $212 \%$ versus voltage-limited charging.

A practical and implementable feedback control scheme particularly well suited to extending battery cell life while yielding maximum performance is model-predictive control (MPC), which is gaining popularity throughout industry in other applications [12]. Employing a "look-ahead" strategy, MPC can foresee dynamic changes before they happen and efficiently compute stepwise-optimal control to achieve a quadratic performance objective. Most importantly however, MPC is able to handle hard (as well as soft) constraints on designated problem variables. The potential employment of MPC for improved cell-level battery control is directly dependent on the availability of certain electrochemical variables internal to the lithium ion cell. Since these variables are not easily (or at all) measurable, they must be estimated from easily obtained voltage-current-temperature information during cell operation. The estimates provided by EKF are highly compatible for use with MPC.

At this point in history, however, the computational complexity of porous-electrode PDE models precludes their use by EKF and real-time control systems. Instead, accurate reduced-order approximate models, which can predict both the cell voltage and internal electrochemical variables, are needed.

For example, Santhanagopalan et al. used single-particle models and simplified porous-electrode models in conjunction with extended and sigma-point Kalman filters to estimate cell SOC [13, 14]. Di Domenico et al. used simplified averaged electrochemical models with both continuous-time linear Kalman filters and extended Kalman filters to estimate cell SOC and particle surface concentration at the current collectors [15, 16]. Neither of these, however, addressed the problem of estimating general internal electrochemical variables at arbitrary points in the cell.

Other efforts similar to this present work include papers by Klein et al., which assumes constant electrolyte concentration and a polynomial approximation to solid concentration to arrive at a partial-differential algebraic model, sampled on a coarse grid across the cell, which is then used by an output error injection state observer [17, 18]. This model is of higher computational complexity than the one we use in this work, and the output error injection observer does not provide confidence bounds on the estimated states. While the authors state that their observer can estimate the values of internal electrochemical variables, they show results of SOC estimation only.

Perhaps the most similar work is that of Smith et al. [10, 19]. These authors simplify the electrochemical model using a transfer-function approach, as do we, which forgoes the necessity of solving PDEs on a spatial grid. However, they do not employ the nonlinear corrections and model blending that characterize the model that we use, and which significantly improve model predictions across a wide range of state-of-charge and temperature, and at high rates. Further, they used a linear Kalman filter on their fully linear model, as opposed to the nonlinear EKF used herein. And, while they state that their methodology can estimate internal electrochemical variables, they do not derive the 
extra steps needed to correctly estimate the variables and the confidence interval of the estimate from the $a$ posteriori model state vector estimate and covariance, and they show results of cell and electrode SOC estimation only.

In this work, we use EKF with a one-dimensional physics-based reduced-order model of lithium-ion dynamics. This five-state discrete-time model is of similar computational complexity to an equivalent-circuit model, and gives very good predictions of cell voltage as well as the cell internal variables. We have presented this model elsewhere in a series of three papers: the first paper introduced the "discrete-time realization algorithm" (DRA) as a subspace projection method for converting a transcendental transfer function into a discrete-time state-space reduced-order model [20]. The second paper showed how to find transcendental transfer functions corresponding to lithium-ion internal cell dynamics [21], and invoked the DRA to create a ROM that is linearized to give accurate predictions around a single SOC and temperature set-point. This ROM was able to predict cell voltage as well as cell potentials $\phi_{s}(x, t)$ and $\phi_{e}(x, t)$, lithium concentrations $c_{s, e}(x, t)$ and $c_{e}(x, t)$, and intercalation flux $j(x, t)$ for any desired set of one-dimensional internal cell locations $x$. The third paper showed how to generalize the method to extend the model's accuracy over a wide range of both SOC and operating temperatures [22] resulting in a very high fidelity nonlinear five-state model.

The purpose of this present paper, then, is to show how to combine this one-dimensional ROM with the EKF algorithm to estimate not only cell SOC but also cell internal electrochemical variables. We believe this is the first result that has been reported on estimating, in real time, cell internal electrochemical variables, and confidence intervals on those estimates, using only a model and measured current, voltage, and temperature data. This methodology opens up possibilities for very sophisticated battery controls to enhance battery pack performance and life using only easily measurable quantities.

The paper is organized as follows. In Section 2, we review the one-dimensional physics-based reduced-order model of cell dynamics upon which this work is based. Section 3, introduces the extended-Kalman-filter algorithm and shows how to generalize the EKF so that its state estimate can yield an estimate of cell internal electrochemical variables. In Section 4, we apply the EKF steps to the physics-based ROM. Results are presented in Section 5. The paper concludes with a summary.

\section{One-dimensional physics-based reduced-order model of cell dynamics}

A first-principles porous-electrode model of lithium-ion cell dynamics was introduced by a number of researchers in the early 1990s [7, 8, 23]. This commonly used model is composed of four coupled nonlinear partial differential equations (PDEs) and one algebraic equation that together describe the dynamics of the internal electrochemical variables of a lithium-ion battery cell:

$$
\begin{aligned}
\frac{\partial c_{s}(r, x, t)}{\partial t} & =\frac{1}{r^{2}} \frac{\partial}{\partial r}\left(D_{s} r^{2} \frac{\partial c_{s}(r, x, t)}{\partial r}\right) \\
\frac{\partial\left(\varepsilon_{e} c_{e}(x, t)\right)}{\partial t} & =\frac{\partial}{\partial x}\left(D_{e}^{\mathrm{eff}} \frac{\partial}{\partial x} c_{e}(x, t)\right)+\left(1-t_{+}^{0}\right) a_{s} j(x, t)
\end{aligned}
$$




$$
\begin{aligned}
a_{s} F j(x, t)= & \frac{\partial}{\partial x}\left(\sigma^{\mathrm{eff}} \frac{\partial}{\partial x} \phi_{s}(x, t)\right) \\
-a_{s} F j(x, t)= & \frac{\partial}{\partial x}\left(\kappa^{\mathrm{eff}} \frac{\partial}{\partial x} \phi_{e}(x, t)\right)+\frac{\partial}{\partial x}\left(\kappa_{D}^{\mathrm{eff}} \frac{\partial}{\partial x} \ln c_{e}(x, t)\right) \\
j(x, t)= & k_{0} c_{e}{ }^{1-\alpha}\left(c_{s, \max }-c_{s, e}\right)^{1-\alpha} c_{s, e}{ }^{\alpha} \times \\
& \left(\exp \left(\frac{(1-\alpha) F}{R T} \eta\right)-\exp \left(-\frac{\alpha F}{R T} \eta\right)\right)
\end{aligned}
$$

These equations predict the following electrode-scale variables: reaction flux $j$, potential in the solid phase $\phi_{s}$, and concentration of lithium in the solid phase $c_{s}$; and also the cell-scale variables: potential in the electrolyte phase $\phi_{e}$ and concentration of lithium in the electrolyte phase $c_{e}$. The boundary and initial conditions, and a description of the parameters used in the equations can be found in Doyle et al. [23]. The model is specialized to a one-dimensional description of cell dynamics with an added radial pseudo-dimension that describes lithium concentration inside the solid particles (resulting in a "pseudo-two-dimensional" model). Figure 1 depicts the geometry of a lithium-ion cell cross section; the model $x$ dimension spans the cell width, while the model $r$ pseudo-dimension is measured outward from a particle center.

[Figure 1 about here.]

Simulations using this full-order model (FOM) provide accurate predictions of the internal dynamics of the highly nonlinear electrochemical variables within the lithium-ion battery cell, but must be implemented using a very sophisticated multi-physics software package. ROMs hope to provide very similar results to those of the FOMs, but with much less computational complexity.

One approach to creating a high-fidelity ROM was proposed by Lee et al. in a series of three papers. In [20], the authors presented a new method to reduce a (possibly transcendental) transfer function to a highly optimized ROM. They called this method the "discrete-time realization algorithm" or DRA. The DRA avoids the difficulties associated with solving an iterative, nonlinear optimization problem by replacing it with a non-iterative, subspacebased algorithm capable of delivering a low-order state-space model without the need for an explicit parameterization of the system model. In [21], the DRA is applied to the problem of creating a ROM of the model of Eqs. (1) to (5), linearized around a single SOC and temperature setpoint, via first finding transcendental transfer functions of the cell internal electrochemical variables. In [22], the ROM is generalized to give accurate predictions over a wide SOC and temperature operating window. The final reduced-order discrete-time state-space realization is of the form

$$
\begin{aligned}
\mathbf{x}_{k+1} & =\mathbf{A}_{k} \mathbf{x}_{k}+\mathbf{B}_{k} i_{\mathrm{app}, k} \\
\tilde{\mathbf{y}}_{k} & =\mathbf{C}_{k} \mathbf{x}_{k}+\mathbf{D}_{k} i_{\mathrm{app}, k} \\
\mathbf{y}_{k} & =g_{k}\left(\tilde{\mathbf{y}}_{k}, i_{\mathrm{app}, k}\right) \\
v_{k} & =g_{v_{k}, k}\left(\tilde{\mathbf{y}}_{k}, i_{\mathrm{app}, k}\right),
\end{aligned}
$$


where Eq. (6) describes the dynamics of the system, and Eq. (7) describes how the linearized output $\tilde{\mathbf{y}}_{k}$ is computed as a linear combination of the states $\mathbf{x}_{k}$ and the cell input current $i_{\text {app }, k}$. The matrices $\mathbf{A}_{k}, \mathbf{B}_{k}, \mathbf{C}_{k}$, and $\mathbf{D}_{k}$ are time-varying, and depend on the cell's present SOC and temperature. Also, since $\tilde{\mathbf{y}}_{k}$ is a function of $\mathbf{x}_{k}$ and $i_{\text {app, } k}$, we sometimes write Eq. (8) as $\mathbf{y}_{k}=g_{k}\left(\mathbf{x}_{k}, i_{\text {app }, k}\right)$.

In Eqs. (6) and (7), the model state $\mathbf{x}_{k}$ is nonphysical, but the vector $\tilde{\mathbf{y}}_{k}$ comprises predictions of any set of linearized electrochemical cell internal variables that the user wishes to monitor. Nonlinear corrections must be applied to a number of the entries in $\tilde{\mathbf{y}}_{k}$ to produce accurate predictions of the true value of those variables. The full nonlinear predictions of the internal electrochemical variables are stored in $\mathbf{y}_{k}$, and are computed from $\tilde{\mathbf{y}}_{k}$ and cell current via the function $g_{k}(\cdot)$, as indicated by Eq. (8). Cell voltage can be computed as one component of $\mathbf{y}_{k}$ via $g_{k}(\cdot)$, but we sometimes wish to think about the calculation of cell voltage separately from the calculation of the internal electrochemical variables, so we denote the sub-function inside vector function $g_{k}(\cdot)$ that calculates cell voltage as $g_{v_{k}, k}(\cdot)$, as indicated by Eq. (9). Details of these nonlinear calculations are reviewed here as they are important in the sequel.

Reaction flux: In the discrete-time model, local reaction flux at spatial location $x$ and time index $k$ is denoted by $j_{k}(x)$. Any component in $\mathbf{y}_{k}$ that corresponds to a prediction of $j_{k}(x)$ may be copied directly from the corresponding component in $\tilde{\mathbf{y}}_{k}$. No nonlinear correction is needed for this internal electrochemical variable.

Solid surface concentration: Lithium concentration in the solid at spatial location $x$ and radial location $r$ within a particle is denoted as $c_{s, k}(r, x)$. Here, we are concerned only with the concentration of lithium at the surface of the particles, as that is what determines reaction rate and cell voltage. The surface concentration is denoted as $c_{s, e, k}(x)$. Components of $\tilde{\mathbf{y}}_{k}$ that calculate a linearized approximation to solid surface concentration at some point in either electrode compute the debiased variable $\tilde{c}_{s, e, k}(x)=c_{s, e, k}(x)-c_{s, 0}$, where $c_{s, 0}$ is the equilibrium solid concentration at the model's linearization setpoint. Components in $\mathbf{y}_{k}$ modeling solid surface concentration can thus be found from the corresponding components of $\tilde{\mathbf{y}}_{k}$ as $c_{s, e, k}(x)=\tilde{c}_{s, e, k}(x)+c_{s, 0}$.

Potential in solid: Solid potential at some location $x$ in either the negative or positive electrode is denoted as $\phi_{s, k}(x)$. No nonlinear corrections are needed for spatial locations in the negative electrode; that is, any component in $\mathbf{y}_{k}$ that corresponds to a prediction of solid potential at a point in the negative electrode may be copied directly from the corresponding component in $\tilde{\mathbf{y}}_{k}$. However, in the positive electrode, the linearized solid-potential calculation in $\tilde{\mathbf{y}}_{k}$ directly computes debiased variable $\tilde{\phi}_{s, k}(x)=\phi_{s, k}(x)-v_{k}$, where $v_{k}$ is the cell's voltage at time index $k$, and can be found as described below. Once cell voltage is calculated, we can then compute components in $\mathbf{y}_{k}$ modeling solid potential at points in the positive electrode from the corresponding components of $\tilde{\mathbf{y}}_{k}$ via $\phi_{s, k}(x)=\tilde{\phi}_{s, k}(x)+v_{k}$.

Potential in electrolyte: Electrolyte potential at some location $x$ is denoted as $\phi_{e, k}(x)$. We define a debiased electrolyte potential that subtracts out the potential at the negative current collector where $x=0: \tilde{\phi}_{e, k}(x)=$ 
$\phi_{e, k}(x)-\phi_{e, k}(0)$. Furthermore, we break up $\tilde{\phi}_{e, k}(x)$ into two parts: $\tilde{\phi}_{e, k}(x)=\left[\tilde{\phi}_{e, k}(x)\right]_{1}+\left[\tilde{\phi}_{e, k}(x)\right]_{2}$. The ROM directly computes $\left[\tilde{\phi}_{e, k}(x)\right]_{1}$ as part of $\tilde{\mathbf{y}}_{k}$, but the nonlinear correction $\left[\tilde{\phi}_{e, k}(x)\right]_{2}$ must be added in order to determine $\tilde{\phi}_{e, k}(x)$, where

$$
\left[\tilde{\phi}_{e, k}(x)\right]_{2}=\frac{2 R T\left(1-t_{+}^{0}\right)}{F} \ln \left(\frac{c_{e, k}(x)}{c_{e, k}(0)}\right),
$$

and where calculation of $c_{e, k}(x)$ is described below. Finally, after some steps described in [21], we find that

$$
\phi_{e, k}(x)=\tilde{\phi}_{e, k}(x)-\left[\tilde{\phi}_{s-e, k}^{\mathrm{neg}}(0)\right]^{*}-U_{\mathrm{ocp}}^{\mathrm{neg}}\left(c_{\mathrm{s}, \mathrm{avg}, k}^{\mathrm{neg}}\right),
$$

where $\left[\tilde{\phi}_{s-e, k}^{\text {neg }}(0)\right]^{*}$ and $c_{\mathrm{s}, \text { avg }, k}^{\text {neg }}$ are terms that can be directly computed as members of $\tilde{\mathbf{y}}_{k}$, and $U_{\text {ocp }}^{\text {neg }}(c)$ is the negative-electrode open-circuit-potential as a function of lithium concentration.

Concentration in electrolyte: Lithium concentration in the electrolyte is denoted $c_{e, k}(x)$. We define a debiased electrolyte concentration that subtracts out the equilibrium concentration: $\tilde{c}_{e, k}(x)=c_{e, k}(x)-c_{e, 0}$. Components of $\tilde{\mathbf{y}}_{k}$ that calculate a linearized approximation of electrolyte concentration at some spatial location compute the debiased variable $\tilde{c}_{e, k}(x)$; components in $\mathbf{y}_{k}$ modeling electrolyte concentration can thus be found from the corresponding components of $\tilde{\mathbf{y}}_{k}$ as $c_{e, k}(x)=\tilde{c}_{e, k}(x)+c_{e, 0}$.

Cell voltage: Finally, cell voltage may be computed via the above cell internal variables as

$$
\begin{aligned}
v_{k}=F & \left.R_{\mathrm{film}}^{\mathrm{pos}} j_{k}^{\mathrm{pos}}\left(L^{\mathrm{tot}}\right)-R_{\mathrm{film}}^{\mathrm{neg}} j_{k}^{\mathrm{neg}}(0)\right]+\left[\tilde{\phi}_{e, k}\left(L^{\mathrm{tot}}\right)\right]_{1} \\
+ & {\left[\eta_{k}^{\mathrm{pos}}\left(L^{\mathrm{tot}}\right)-\eta_{k}^{\mathrm{neg}}(0)\right]+\left[\tilde{\phi}_{e, 2}\left(L^{\mathrm{tot}}\right)\right]_{2} } \\
+ & {\left[U_{\mathrm{ocp}}^{\mathrm{pos}}\left(c_{s, e, k}^{\mathrm{pos}}\left(L^{\mathrm{tot}}\right)\right)-U_{\mathrm{ocp}}^{\mathrm{neg}}\left(c_{s, e, k}^{\mathrm{neg}}(0)\right)\right], }
\end{aligned}
$$

where $L^{\text {tot }}$ is the $x$-location of the positive current collector, and

$$
\begin{gathered}
\eta_{k}^{\mathrm{pos}}(x)=F R_{\mathrm{ct}, k}^{\mathrm{pos}}(x) j_{k}^{\mathrm{pos}}(x) \\
\eta_{k}^{\mathrm{neg}}(x)=F R_{\mathrm{ct}, k}^{\mathrm{neg}}(x) j_{k}^{\mathrm{neg}}(x),
\end{gathered}
$$

and $R_{\mathrm{ct}, k}$ is the charge-transfer resistance of the noted electrode, which is calculated as

$$
R_{\mathrm{ct}, k}(x)=\frac{R T}{j_{0, k}(x) F^{2}}
$$

where

$$
j_{0, k}(x)=k_{0}\left(c_{e, k}(x)\right)^{1-\alpha}\left(c_{s, \max }-c_{s, e, k}(x)\right)^{1-\alpha}\left(c_{s, e, k}(x)\right)^{\alpha} .
$$

\section{The extended Kalman filter}

All varieties of Kalman filter require a mathematical model of the system whose internal state is to be estimated. An assumed general, and possibly nonlinear, discrete-time model takes the form

$$
\begin{array}{r}
\mathbf{x}_{k+1}=f_{k}\left(\mathbf{x}_{k}, \mathbf{u}_{k}, \mathbf{w}_{k}\right) \\
\mathbf{z}_{k}=h_{k}\left(\mathbf{x}_{k}, \mathbf{u}_{k}, \mathbf{v}_{k}\right),
\end{array}
$$


where $\mathbf{u}_{k}$ is a known (deterministic/measured) input signal, $\mathbf{x}_{k}$ is the model state vector, $\mathbf{z}_{k}$ is the measurable model output, $\mathbf{w}_{k}$ is a process-noise random input, and $\mathbf{v}_{k}$ is a sensor-noise random input, not to be confused with cell terminal voltage $v_{k}$. In the ROM, $\mathbf{u}_{k}$ is cell input current $i_{\text {app }, k}$ and $\mathbf{z}_{k}$ is cell terminal voltage $v_{k}$.

In a Kalman filter, the present state $\mathbf{x}_{k}$ is estimated using all past and present output measurements $\mathbf{z}_{k}$. These observed output measurements provide feedback that allows the filter to estimate what is happening inside the true system, producing a "minimum mean squared error" (MMSE) estimate of the state, which computes the conditional expectation

$$
\hat{\mathbf{x}}_{k}^{+}=\mathbb{E}\left[\mathbf{x}_{k} \mid \mathbb{Z}_{k}\right]
$$

where $\mathbb{Z}_{k}=\left\{\mathbf{z}_{i}: 0 \leq i \leq k\right\}$ is the set of all measurements from time 0 to time $k$. A general "probabilistic inference" solution recursively computes $\hat{\mathbf{x}}_{k}^{+}$in two major steps—-state prediction and state correction-each having three substeps, assuming that all uncertain variables have a Gaussian distribution [4]. ${ }^{2}$

- General step la: State prediction time update. In this step, an updated prediction of the present value of $\mathbf{x}_{k}$ is computed based on a priori information along with the system model.

$$
\hat{\mathbf{x}}_{k}^{-}=\mathbb{E}\left[\mathbf{x}_{k} \mid \mathbb{Z}_{k-1}\right]=\mathbb{E}\left[f_{k-1}\left(\mathbf{x}_{k-1}, \mathbf{u}_{k-1}, \mathbf{w}_{k-1}\right) \mid \mathbb{Z}_{k-1}\right]
$$

- General step 1b: Error covariance time update. In this step, the predicted state-estimate error covariance matrix $\Sigma_{\tilde{\mathbf{x}}, k}^{-}$is determined based on a priori information along with the system model.

$$
\Sigma_{\tilde{\mathbf{x}}, k}^{-}=\mathbb{E}\left[\left(\tilde{\mathbf{x}}_{k}^{-}\right)\left(\tilde{\mathbf{x}}_{k}^{-}\right)^{T}\right]
$$

where $\tilde{\mathbf{x}}_{k}^{-}=\mathbf{x}_{k}-\hat{\mathbf{x}}_{k}^{-}$.

- General step 1c: Predict system output. In this step, the system's output is predicted using a priori information.

$$
\hat{\mathbf{z}}_{k}=\mathbb{E}\left[\mathbf{z}_{k} \mid \mathbb{Z}_{k-1}\right]=\mathbb{E}\left[h_{k}\left(\mathbf{x}_{k}, \mathbf{u}_{k}, \mathbf{v}_{k}\right) \mid \mathbb{Z}_{k-1}\right]
$$

- General step 2a: Estimator Kalman gain matrix. In this step, the estimator gain matrix is computed.

$$
\mathbf{L}_{k}=\Sigma_{\tilde{\mathbf{z}}, k}^{-} \Sigma_{\tilde{\mathbf{z}}, k}^{-1},
$$

where $\Sigma_{\tilde{\mathbf{x}} \tilde{\mathbf{z}}, k}^{-}$is cross-covariance between $\tilde{\mathbf{x}}$ and $\tilde{\mathbf{z}}$ and $\Sigma_{\tilde{\mathbf{z}}, k}^{-1}$ is the inverse of the autocovariance of $\tilde{\mathbf{z}}$.

- General step 2b: State estimate measurement update. In this step, a posteriori state estimate is computed by updating a priori estimate using the estimator gain and the output prediction error, $\tilde{\mathbf{z}}_{k}=\mathbf{z}_{k}-\hat{\mathbf{z}}_{k}$.

$$
\hat{\mathbf{x}}_{k}^{+}=\hat{\mathbf{x}}_{k}^{-}+\mathbf{L}_{k}\left(\mathbf{z}_{k}-\hat{\mathbf{z}}_{k}\right) \text {. }
$$

\footnotetext{
${ }^{2}$ In this paper, the "hat" decoration (.) on a mathematical symbol indicates a prediction or estimate of the quantity under the decoration, a "tilde" decoration (.) indicates either a prediction or estimation error of that quantity or that the quantity is a linearized version of a nonlinear variable, a "plus" (+) superscript indicates an estimate (i.e., a computation that is based on all possible measurable data up until this point), and a "minus" (-) superscript indicates a prediction (i.e., a computation that is based on previously collected data, not including the presently measurable data).
} 
- General step 2c: Error covariance measurement update. In this final step, the error covariance matrix is updated as the measurement update has decreased the uncertainty in the state estimate.

$$
\Sigma_{\tilde{\mathbf{x}}, k}^{+}=\mathbb{E}\left[\left(\tilde{\mathbf{x}}_{k}^{+}\right)\left(\tilde{\mathbf{x}}_{k}^{+}\right)^{T}\right]=\Sigma_{\tilde{\mathbf{x}}, k}^{-}-\mathbf{L}_{k} \Sigma_{\tilde{\mathbf{z}}, k} \mathbf{L}_{k}^{T}
$$

When the system model is linear and the noises are additive, white, and Gaussian, the general sequential probabilistic inference steps just outlined may be evaluated exactly in closed form, resulting in the linear Kalman filter. When the system model is nonlinear, these steps must be approximated instead. For the EKF, this is done via analytic linearization of the model at each point in time.

Because of its basis in sequential probabilistic inference, the EKF can be also be described by two main components, predication and correction, both of which contain three sub-steps each. The prediction component updates the state prediction and corresponding error covariance, and finally predict the system output. The correction component calculates the Kalman gain and calculates measurement updates for the state estimate and corresponding error covariance.

Two simplifying assumptions are made by the EKF when the general sequential inference equations are adapted to a nonlinear system:

1. The EKF assumes that $\mathbb{E}[\mathrm{fn}(\mathbf{x})] \approx \mathrm{fn}(\mathbb{E}[\mathbf{x}])$, when the state estimates are computed.

2. The EKF employs Taylor-series expansion approximation to linearize the system equations around the present operating point, when the covariance estimates are computed.

Applying these two assumptions gives the following six steps:

- EKF step 1a: State prediction time update. In this step, the expected value of the new state is approximated by the assumption that it is reasonable to simply propagate $\hat{\mathbf{x}}_{k-1}^{+}$and $\overline{\mathbf{w}}_{k-1}$ through the state equation as

$$
\begin{aligned}
\hat{\mathbf{x}}_{k}^{-} & =\mathbb{E}\left[f_{k-1}\left(\mathbf{x}_{k-1}, \mathbf{u}_{k-1}, \mathbf{w}_{k-1}\right) \mid \mathbb{Z}_{k-1}\right] \\
& \approx f_{k-1}\left(\hat{\mathbf{x}}_{k-1}^{+}, \mathbf{u}_{k-1}, \overline{\mathbf{w}}_{k-1}\right) .
\end{aligned}
$$

- EKF step 1b: Error covariance time update. In this step, the covariance prediction is accomplished by providing an approximation for $\tilde{\mathbf{x}}_{k}^{-}$. That is to define $\tilde{\mathbf{x}}_{k}^{-}=\mathbf{x}_{k}-\hat{\mathbf{x}}_{k}^{-}$, which leads to $\tilde{\mathbf{x}}_{k}^{-}=f_{k-1}\left(\mathbf{x}_{k-1}, \mathbf{u}_{k-1}, \mathbf{w}_{k-1}\right)-$ $f_{k-1}\left(\hat{\mathbf{x}}_{k-1}^{+}, \mathbf{u}_{k-1}, \overline{\mathbf{w}}_{k-1}\right)$. The second term is then approximated using a Taylor series expansion around the prior operating point. Through some substitution, the predicted covariance is found as

$$
\Sigma_{\tilde{\mathbf{x}}, k}^{-}=\mathbb{E}\left[\left(\tilde{\mathbf{x}}_{k}^{-}\right)\left(\tilde{\mathbf{x}}_{k}^{-}\right)^{T}\right] \approx \hat{\mathbf{A}}_{k-1} \Sigma_{\tilde{\mathbf{x}}, k-1}^{+} \hat{\mathbf{A}}_{k-1}^{T}+\hat{\mathbf{B}}_{k-1} \Sigma_{\widetilde{\mathbf{w}}} \hat{\mathbf{B}}_{k-1}^{T},
$$

where

$$
\hat{\mathbf{A}}_{k}=\left.\frac{\mathrm{d} f_{k}\left(\mathbf{x}_{k}, \mathbf{u}_{k}, \mathbf{w}_{k}\right)}{\mathrm{d} \mathbf{x}_{k}}\right|_{\mathbf{x}_{k}=\hat{\mathbf{x}}_{k}^{+}} \text {and } \hat{\mathbf{B}}_{k}=\left.\frac{\mathrm{d} f_{k}\left(\mathbf{x}_{k}, \mathbf{u}_{k}, \mathbf{w}_{k}\right)}{\mathrm{d} \mathbf{w}_{k}}\right|_{\mathbf{w}_{k}=\overline{\mathbf{w}}_{k}} .
$$


- EKF step 1c: Predict system output. In this step, the system output is estimated by the assumption that propagating $\hat{\mathbf{x}}_{k}^{-}$and the mean sensor noise is the best approximation that can be established. This applies to the system output equation as

$$
\hat{\mathbf{z}}_{k}=\mathbb{E}\left[h_{k}\left(\mathbf{x}_{k}, \mathbf{u}_{k}, \mathbf{v}_{k}\right) \mid \mathbb{Z}_{k-1}\right] \approx h_{k}\left(\hat{\mathbf{x}}_{k}^{-}, \mathbf{u}_{k}, \overline{\mathbf{v}}_{k}\right),
$$

- EKF step 2a: Estimator Kalman gain matrix. The Kalman gain $\mathbf{L}_{k}$ is calculated by defining the output prediction error as $\tilde{\mathbf{z}}_{k}=\mathbf{z}_{k}-\hat{\mathbf{z}}_{k}$ and deriving an approximation using the same procedures of step 1b, Taylor series expansion using total differentials. This leads to the equation for the Kalman gain as

$$
\mathbf{L}_{k}=\Sigma_{\tilde{\mathbf{x}}, k}^{-} \hat{\mathbf{C}}_{k}^{T}\left[\hat{\mathbf{C}}_{k} \Sigma_{\tilde{\mathbf{x}}, k}^{-} \hat{\mathbf{C}}_{k}^{T}+\hat{\mathbf{D}}_{k} \Sigma_{\tilde{\mathbf{v}}} \hat{\mathbf{D}}_{k}^{T}\right]^{-1}
$$

where

$$
\hat{\mathbf{C}}_{k}=\left.\frac{\mathrm{d} h_{k}\left(\mathbf{x}_{k}, \mathbf{u}_{k}, \mathbf{v}_{k}\right)}{\mathrm{d} \mathbf{x}_{k}}\right|_{\mathbf{x}_{k}=\hat{\mathbf{x}}_{k}^{-}} \text {and } \hat{\mathbf{D}}_{k}=\left.\frac{\mathrm{d} h_{k}\left(\mathbf{x}_{k}, \mathbf{u}_{k}, \mathbf{v}_{k}\right)}{\mathrm{d} \mathbf{v}_{k}}\right|_{\mathbf{v}_{k}=\overline{\mathbf{v}}_{k}} .
$$

- EKF step 2b: State estimate measurement update. This probabilistic inference step is implemented exactly by EKF (i.e., no approximations are made),

$$
\hat{\mathbf{x}}_{k}^{+}=\hat{\mathbf{x}}_{k}^{-}+\mathbf{L}_{k}\left(\mathbf{z}_{k}-\hat{\mathbf{z}}_{k}\right) .
$$

- EKF step 2c: Error covariance measurement update. This step is also implemented exactly by EKF,

$$
\Sigma_{\tilde{\mathbf{x}}, k}^{+}=\Sigma_{\tilde{\mathbf{x}}, k}^{-}-\mathbf{L}_{k} \Sigma_{\tilde{\mathbf{z}}, k} \mathbf{L}_{k}^{T}
$$

These steps for the standard EKF are sufficient if the objective is simply to estimate the (nonphysical) state $\mathbf{x}_{k}$ of the cell ROM. In our case, however, we are more interested in estimating the cell internal electrochemical variables, which are computed from the state as described by Eqs. (7) and (8), and by the details for the nonlinear corrections explained in Section 2. Therefore, we must add a third step following the existing prediction/correction steps of the EKF.

The nonlinear electrochemical states are calculated from Eqs. (7) and (8) by employing EKF assumption 1

$$
\hat{\mathbf{y}}_{k}=\left.g_{k}\left(\mathbf{x}_{k}, i_{\mathrm{app}, k}\right)\right|_{\mathbf{x}_{k}=\hat{\mathbf{x}}_{k}^{+}} .
$$

The covariance for the electrochemical states is computed via Taylor-series expansion and EKF assumption 2 as

$$
\begin{aligned}
\Sigma_{\tilde{\mathbf{y}}, k} & =\mathbb{E}\left[\left(\mathbf{y}_{k}-\hat{\mathbf{y}}_{k}\right)\left(\mathbf{y}_{k}-\hat{\mathbf{y}}_{k}\right)^{T}\right] \\
& \approx\left(\hat{\mathbf{C}}_{k}^{+}\right)^{T} \Sigma_{\tilde{\mathbf{x}}, k}\left(\hat{\mathbf{C}}_{k}^{+}\right),
\end{aligned}
$$

where

$$
\hat{\mathbf{C}}_{k}^{+}=\left.\frac{\mathrm{d} g_{k}\left(\mathbf{x}_{k}, i_{\mathrm{app}, k}\right)}{\mathrm{d} \mathbf{x}_{k}}\right|_{\mathbf{x}_{k}=\hat{\mathbf{x}}_{k}^{+}} .
$$

Table 1 summarizes the EKF steps in a convenient format for quick reference. 
Note that because of numeric error, it is possible in an implementation for the covariance matrices to become negative definite, which contradicts theory. We can correct for this by computing the "nearest" positive semi-definite covariance matrix every time a covariance matrix is updated [24]. This is done by first factoring the covariance matrix of interest into its singular-value decomposition

$$
\Sigma=U \Lambda V^{T}
$$

then computing $H=V \Lambda V^{T}$, and finally replacing the original covariance matrix by

$$
\Sigma:=\frac{\Sigma+\Sigma^{T}+H+H^{T}}{4} .
$$

[Table 1 about here.]

\section{Applying the EKF to the DRA-Based ROM}

In this section, we show how the general EKF steps from Table 1 can be applied to the DRA-based ROM.

EKF step 1a. To implement this step, we require a definition of the model state equation. To do so, we modify Eq. (6) to be

$$
\mathbf{x}_{k}=\mathbf{A}_{k-1} \mathbf{x}_{k-1}+\mathbf{B}_{k-1}\left(i_{\mathrm{app}, k-1}+\mathbf{w}_{k-1}\right),
$$

where the process noise is modeled as being additive to the cell input current. Therefore,

$$
f_{k-1}\left(\mathbf{x}_{k-1}, i_{\text {app }, k-1}, \mathbf{w}_{k-1}\right)=\mathbf{A}_{k-1} \mathbf{x}_{k-1}+\mathbf{B}_{k-1}\left(i_{\text {app }, k-1}+\mathbf{w}_{k-1}\right) .
$$

To find $\mathbf{A}_{k-1}$ and $\mathbf{B}_{k-1}$, the cell state of charge is computed from $\mathbf{x}_{k-1}^{+}$, and cell temperature is either measured or computed from the system state via a thermal model. Model blending, as described in [22] provides the values of these time-varying matrices by employing bilinear interpolation on the corresponding matrices from the stored precomputed models created at the four nearest linearization setpoints.

EKF step 1b. To implement this step, we require $\hat{\mathbf{A}}_{k-1}^{+}$and $\hat{\mathbf{B}}_{k-1}$. Examining Eq. (24), we determine that $\hat{\mathbf{A}}_{k-1}^{+}=\mathbf{A}_{k-1}$ and $\hat{\mathbf{B}}_{k-1}=\mathbf{B}_{k-1}$.

EKF step 1c. To implement this step, we require a definition of the model output equation. To do so, we have modified Eq. (9) to be

$$
v_{k}=\mathbf{z}_{k}=g_{v_{k}, k}\left(\mathbf{y}_{k}, i_{\text {app }, k}\right)+\mathbf{v}_{k},
$$

where the measurement noise is modeled as being additive to the voltage sensor. Therefore,

$$
h_{k}\left(\mathbf{x}_{k}, i_{\mathrm{app}, k}, \mathbf{v}_{k}\right)=g_{v_{k}, k}\left(\mathbf{x}_{k}, i_{\mathrm{app}, k}\right)+\mathbf{v}_{k},
$$

where the computations implemented by $g_{v_{k}, k}\left(\mathbf{x}_{k}, i_{\text {app }, k}\right)$ are described at the end of Section 2. 
EKF step $2 a$. To implement this step, we require $\hat{\mathbf{C}}_{k}^{-}$and $\hat{\mathbf{D}}_{k}$. Examining Eq. (25), we determine that $\hat{\mathbf{D}}_{k}=I$. However, calculation of $\hat{\mathbf{C}}_{k}^{-}$is more complicated. To compute the result we desire here, and similar results for EKF step $3 b$, we will need to be able to calculate $\mathrm{d} g_{k}\left(\mathbf{x}_{k}, i_{\text {app }, k}\right) / \mathrm{d} \mathbf{x}_{k}$ for all of the internal electrochemical variables of interest. We look at these computations here.

Reaction flux: Components of $g_{k}\left(\mathbf{x}_{k}, i_{\mathrm{app}, k}\right)$ corresponding to a computation of reaction flux at some spatial location $x$ are computed by Eq. (7) as

$$
j_{k}(x)=\mathbf{C}_{j x, k} \mathbf{x}_{k}+\mathbf{D}_{j x, k} i_{\text {app }, k}
$$

where $\mathbf{C}_{j x, k}$ is the row in $\mathbf{C}_{k}$ corresponding to the computation of $j_{k}(x)$ and $\mathbf{D}_{j x, k}$ is the row in $\mathbf{D}_{k}$ corresponding to the computation of $j_{k}(x)$. Therefore, $\mathrm{d} j_{k}(x) / \mathrm{d} \mathbf{x}_{k}=\mathbf{C}_{j x, k}$.

Solid surface concentration: Components of $g_{k}\left(\mathbf{x}_{k}, i_{\mathrm{app}, k}\right)$ corresponding to a computation of lithium solid surface concentration at some spatial location $x$ are computed by Eq. (7) as

$$
c_{s, e, k}(x)=\mathbf{C}_{c s e x, k} \mathbf{x}_{k}+\mathbf{D}_{c \operatorname{sex}, k} i_{\mathrm{app}, k}+c_{s, 0}
$$

where $\mathbf{C}_{c s e x, k}$ is the row in $\mathbf{C}_{k}$ corresponding to the computation of $c_{s, e, k}(x)$ and $\mathbf{D}_{c s e x, k}$ is the row in $\mathbf{D}_{k}$ corresponding to the computation of $c_{s, e, k}(x)$. Therefore, $\mathrm{d} c_{s, e, k}(x) / \mathrm{d} \mathbf{x}_{k}=\mathbf{C}_{c s e x, k}$.

Potential in solid: Components of $g_{k}\left(\mathbf{x}_{k}, i_{\text {app }, k}\right)$ corresponding to a computation of solid potential at some spatial location $x$ in the negative electrode are computed by Eq. (7) as

$$
\phi_{s, k}(x)=\mathbf{C}_{\phi_{s} x, k} \mathbf{x}_{k}+\mathbf{D}_{\phi_{s} x, k} i_{\mathrm{app}, k}
$$

where $\mathbf{C}_{\phi_{s} x, k}$ is the row in $\mathbf{C}_{k}$ corresponding to the computation of $\phi_{s, k}(x)$ and $\mathbf{D}_{\phi_{s} x, k}$ is the row in $\mathbf{D}_{k}$ corresponding to the computation of $\phi_{s, k}(x)$. Therefore, for negative-electrode locations, $\mathrm{d} \phi_{s, k}(x) / \mathrm{d} \mathbf{x}_{k}=\mathbf{C}_{\phi_{s} x, k}$.

Components of $g_{k}\left(\mathbf{x}_{k}, i_{\text {app }, k}\right)$ corresponding to a computation of solid potential at some spatial location $x$ in the positive electrode are computed by Eq. (7) as

$$
\phi_{s, k}(x)=\mathbf{C}_{\phi_{s} x, k} \mathbf{x}_{k}+\mathbf{D}_{\phi_{s} x, k} i_{\mathrm{app}, k}+v_{k}
$$

where $\mathbf{C}_{\phi_{s} x, k}$ is the row in $\mathbf{C}_{k}$ corresponding to the computation of $\phi_{s, k}(x), \mathbf{D}_{\phi_{s} x, k}$ is the row in $\mathbf{D}_{k}$ corresponding to the computation of $\phi_{s, k}(x)$, and $v_{k}$ is cell voltage. Therefore, for positive-electrode locations, $\mathrm{d} \phi_{s, k}(x) / \mathrm{d} \mathbf{x}_{k}=$ $\mathbf{C}_{\phi_{s} x, k}+\mathrm{d} v_{k} / \mathrm{d} \mathbf{x}_{k}$. The derivatives of cell voltage $v_{k}$ with respect to state and input are described later in this section.

Concentration in electrolyte: Components of $g_{k}\left(\mathbf{x}_{k}, i_{\mathrm{app}, k}\right)$ corresponding to a computation of lithium electrolyte concentration at some spatial location $x$ are computed by Eq. (7) as

$$
c_{e, k}(x)=\mathbf{C}_{c e x, k} \mathbf{x}_{k}+\mathbf{D}_{c e x, k} i_{\mathrm{app}, k}+c_{e, 0}
$$


where $\mathbf{C}_{c e x, k}$ is the row in $\mathbf{C}_{k}$ corresponding to the computation of $c_{e, k}(x)$ and $\mathbf{D}_{c e x, k}$ is the row in $\mathbf{D}_{k}$ corresponding to the computation of $c_{e, k}(x)$. Therefore, $\mathrm{d} c_{e, k}(x) / \mathrm{d} \mathbf{x}_{k}=\mathbf{C}_{c e x, k}$.

Potential in electrolyte: Components of $g_{k}\left(\mathbf{x}_{k}, i_{\mathrm{app}, k}\right)$ corresponding to a computation of electrolyte potential at some spatial location $x$ are computed by Eq. (7) in four parts:

$$
\begin{gathered}
\phi_{e, k}(x)=\left[\tilde{\phi}_{e, k}(x)\right]_{1}+\frac{2 R T\left(1-t_{+}^{0}\right)}{F} \ln \left(\frac{c_{e, k}(x)}{c_{e, k}(0)}\right) \\
-\left[\tilde{\phi}_{s-e, k}^{\mathrm{neg}}(0)\right]^{*}-U_{\mathrm{ocp}}^{\mathrm{neg}}\left(c_{\mathrm{s}, \mathrm{avg}, k}^{\mathrm{neg}}\right) .
\end{gathered}
$$

The individual components of this equation that have not yet been described are computed as

$$
\begin{aligned}
{\left[\tilde{\phi}_{e, k}(x)\right]_{1} } & =\mathbf{C}_{\tilde{\phi}_{e l} x, k} \mathbf{x}_{k}+\mathbf{D}_{\tilde{\phi}_{e l} x, k} i_{\mathrm{app}, k} \\
{\left[\tilde{\phi}_{s-e, k}^{\text {neg }}(0)\right]^{*} } & =\mathbf{C}_{\tilde{\phi}_{s e l}^{*} 0, k} \mathbf{x}_{k}+\mathbf{D}_{\tilde{\phi}_{s e}^{*} 0, k} i_{\mathrm{app}, k} \\
c_{\mathrm{s}, \mathrm{avg}, k}^{\text {neg }} & =\mathbf{C}_{\overline{c s, k}, \mathrm{neg}}^{\text {neg }} \mathbf{x}_{k}+\mathbf{D}_{\overline{c s, k}, k}^{\mathrm{neg}} i_{\mathrm{app}, k},
\end{aligned}
$$

where the subscript notation on the $\mathbf{C}$ and $\mathbf{D}$ matrices carry the same idea as used the rest of this section. Therefore,

$$
\begin{aligned}
\frac{\mathrm{d} \phi_{e, k}(x)}{\mathrm{d} \mathbf{x}_{k}}=\mathbf{C}_{\tilde{\phi}_{e l} x, k}-\mathbf{C}_{\tilde{\phi}_{s-e}^{*} 0, k} \\
+\frac{2 R T\left(1-t_{+}^{0}\right)}{F}\left[\frac{\mathbf{C}_{c e x, k}}{c_{e, k}(x)}-\frac{\mathbf{C}_{c e 0, k}}{c_{e, k}(0)}\right] \\
\quad-\left.\frac{\mathrm{d} U_{\mathrm{ocp}}^{\mathrm{neg}}\left(c_{s}\right)}{\mathrm{d} c_{s}}\right|_{c_{s}=c_{s, a v g, k}^{\mathrm{neg}}} \mathbf{C}_{\overline{c s}, k}^{\mathrm{neg}} .
\end{aligned}
$$

Cell voltage: When computing derivatives of cell voltage, we approximate $R_{\mathrm{ct}, k}(x)$ as being independent of $\mathbf{x}_{k}$. Therefore, using Eq. (10),

$$
\begin{aligned}
\frac{\mathrm{d} v_{k}}{\mathrm{~d} \mathbf{x}_{k}}=F( & \left.R_{\mathrm{film}}^{\mathrm{pos}}+R_{\mathrm{ct}, k}^{\mathrm{pos}}\left(L^{\mathrm{tot}}\right)\right) \mathbf{C}_{j L^{\mathrm{tot}, k}} \\
& -F\left(R_{\mathrm{film}}^{\mathrm{neg}}+R_{\mathrm{ct}, k}^{\mathrm{neg}}(0)\right) \mathbf{C}_{j 0, k}+\mathbf{C}_{\tilde{\phi}_{e l} L^{\mathrm{to},}, k} \\
& +\frac{2 R T\left(1-t_{+}^{0}\right)}{F}\left[\frac{\mathbf{C}_{c e L^{\mathrm{tot}}, k}}{c_{e, k}\left(L^{\mathrm{tot}}\right)}-\frac{\mathbf{C}_{c e 0, k}}{c_{e, k}(0)}\right] \\
& +\left.\frac{\mathrm{d} U_{\mathrm{ocp}}^{\mathrm{pos}}\left(c_{s}\right)}{\mathrm{d} c_{s}}\right|_{c_{s}=c_{s, e, k}^{\mathrm{pos}}\left(L^{\mathrm{tot}}\right)} \mathbf{C}_{c s e L^{\mathrm{tot}, k}}^{\mathrm{pos}} \\
& -\left.\frac{\mathrm{d} U_{\mathrm{ocp}}^{\mathrm{neg}}\left(c_{s}\right)}{\mathrm{d} c_{s}}\right|_{c_{s}=c_{s, e, k}^{\mathrm{neg}}(0)} \mathbf{C}_{c s e 0, k}^{\mathrm{neg}} .
\end{aligned}
$$

So, $\hat{\mathbf{C}}_{k}^{-}$is found as $\mathrm{d} v_{k} / \mathrm{d} \mathbf{x}_{k}$ when the constants in the calculation (particularly the $\mathbf{C}_{k}$ matrix rows) are found using the state of the model evaluated at $\hat{\mathbf{x}}_{k}^{-}$.

EKF steps $2 b, 2 c$, and $3 a$. These steps can be evaluated using the variables already defined, where the computations implemented by $g_{k}\left(\mathbf{x}_{k}, i_{\text {app }, k}\right)$ are described at the end of Section 2. 
EKF step $3 b$. In this step, $\hat{\mathbf{C}}_{k}^{+}$is found as described for "EKF step $2 \mathrm{a}$ " for the internal electrochemical variables of interest when the constants in the calculation (particularly the $\mathbf{C}_{k}$ matrix rows) are found using the state of the model evaluated at $\hat{\mathbf{x}}_{k}^{+}$. This generally requires re-blending the $\mathbf{C}_{k}$ matrix based on the update to the cell state-of-charge estimate between $\hat{\mathbf{x}}_{k}^{-}$and $\hat{\mathbf{x}}_{k}^{+}$.

\section{EKF simulation and results}

We now present some results to demonstrate the effectiveness of an EKF used to estimate the state of charge and internal electrochemical variables of a cell under load. We chose to use COMSOL Multiphysics full-order model simulations of Eqs. (1) through (5) as the desired output of the estimator, since "truth values" for all electrochemical variables are readily available from the simulator. It is beyond the scope of this present work to validate filter operation against values measured from a physical cell in an instrumented laboratory setup.

The cell under consideration is described by the parameters in [23], which are listed in Table 2. The EKF used a discrete-time fifth-order ROM as its basis, generated by the DRA for every SOC between $0 \%$ and $100 \%$ in $20 \%$ increments, for an assumed constant temperature of $25^{\circ} \mathrm{C}$. As discussed in [21], the ROM-generation process is entirely automated and hands free. The final ROM used model blending, as described in [22].

[Table 2 about here.]

The cell input current for the simulations was based on the Environmental Protection Agency's (EPA) Urban Dynamometer Driving Schedule (UDDS), which was developed to represent city driving conditions for light duty vehicles. Figure 2 shows the normalized UDDS profile of current versus time, as computed for a medium-size electric vehicle. The maximum absolute rate is $2 \mathrm{C}$, which for this cell corresponds to $41 \mathrm{~A}$. The overall profile was constrained to be charge neutral, as described in the results section of [22]. In all simulations, the truth data was generated from a FOM simulation that was initialized to correspond to a cell in electrochemical equilibrium at a $60 \%$ state of charge.

[Figure 2 about here.]

As the true cell was known to be at rest prior to the start of simulation, the EKF initial state was set to $\hat{\mathbf{x}}_{0}^{+}=0$. The initial covariance was set to

$$
\Sigma_{\tilde{\mathbf{x}}, 0}^{+}=\operatorname{diag}\left[10^{3}, 10^{3}, 10^{3}, 10^{3}, 10^{12}\right]
$$

where the first four components of $\mathbf{x}_{k}$ are general-purpose states, and the final component is the integrator state. Higher initial uncertainty is applied to the integrator state, which is used in the ROM calculations of solid concentration (and hence state of charge), to allow the EKF to recover from a faulty initialization (as described in Section 5.2). The noise-covariance matrices were chosen to be $\Sigma_{\widetilde{\mathbf{w}}}=0.1$ and $\Sigma_{\tilde{\mathbf{v}}}=0.1$, and noise means were modeled as $\overline{\mathbf{w}}=\overline{\mathbf{v}}=0$, which allowed the EKF to give good estimates even though the ROM is not a perfect match to the FOM. 


\subsection{Correct initialization of EKF}

In the first set of simulations, the EKF was initialized correctly, as described. State-of-charge estimation results are presented in Fig. 3. These plots have some features that are common with other results that we present, so we discuss those here.

In Frame (a) on the left, the thick solid black line shows the truth result, calculated by the COMSOL Multiphysics simulation of Eqs. (1) through (5) for the cell input current in Fig. 2. The thick dashed gray line shows the state-ofcharge estimate produced by the EKF. The thin gray lines show the "three sigma" error bounds on the state-of-charge estimate, as calculated by the EKF from $\Sigma_{\tilde{\mathbf{x}}, k}^{+}$. If the EKF is working properly, then the truth should reside within these error bounds $99 \%$ of the time. In this case, the truth is always within the error bounds, which allows the estimate to be used with confidence.

In Frame (b), the thick solid line shows the estimation error. This is computed as truth value minus estimated value. In this example, we see maximum absolute error on the order of $0.1 \%$. Later figures of this type will also show thin solid lines to indicate the error bounds of the estimate. If the EKF is working properly, then the zero point should reside within these error bounds $99 \%$ of the time. Again, this is true for the SOC estimate (the thin solid lines are not visible as the vertical scale for this plot was chosen to zoom in on detail rather than to show the correctness of the error bounds, which had already been established in Frame (a) of the figure).

[Figure 3 about here.]

Estimation results for internal electrochemical variable $j_{k}(x)$ are presented in Fig. 4. It is not possible to show outcomes for every $x$ location in the cell, so we have chosen to limit the plots to display results at the negativeelectrode/separator boundary and at the positive-electrode/separator boundary, as the internal cell electrochemical variables tend to experience the greatest dynamic fluctuation at these positions and hence these are the most difficult locations at which to make good estimates. (We follow the same pattern for all other cell internal electrochemical variable results presented in this paper.)

In Frames (a) and (c), we see that the EKF estimates do tend to follow the truth values quite well, but it is difficult to see whether the error bounds are correct. In Frames (b) and (d), it is easier to judge the scale of the estimation error, but it is still difficult to see how frequently the estimation error bounds encompass zero error. It turns out that the EKF error bounds on this variable are far too tight: they surround zero only about $15 \%$ of the time for the negative-electrode variable and only about $2 \%$ of the time for the positive-electrode variable, whereas theory predicts that they should encompass zero $99 \%$ of the time. So, while the estimates are quite good, the error bounds cannot be trusted.

[Figure 4 about here.]

Estimation results for internal electrochemical variable $\phi_{s, k}(x)$ are presented in Fig. 5. In Frames (a) and (c), we see that the EKF estimates tend to follow the truth values quite well, but it is difficult in (a) to see whether the error bounds are correct. In Frames (b) and (d), it is easier to judge the scale of the estimation error, but in (b) it is still difficult 
to see how frequently the estimation error bounds surround the zero value. It turns out that the EKF error bounds on this variable are again too tight: they surround zero only about $35 \%$ of the time (the error bounds are correct for the positive-electrode variable $100 \%$ of the time). So, estimates for both electrodes are quite good, but the error bounds for negative-electrode estimates cannot be trusted.

[Figure 5 about here.]

Estimation results for internal electrochemical variables $\phi_{e, k}(x)$ and $c_{s, e, k}(x)$ are presented in Figs. 6 and 7, respectively. The plots in these figures are organized as before. For these variables, we see good estimates with error bounds that are trustworthy in both electrodes.

[Figure 6 about here.]

[Figure 7 about here.]

Finally, estimation results for internal electrochemical variable $c_{e, k}(x)$ are presented in Fig. 8. Estimates and bounds are generally trustworthy in both electrodes (the bounds are in error about $2 \%$ of the time, which is close to what would be predicted by theory).

[Figure 8 about here.]

\subsection{Incorrect initialization of EKF}

In an embedded application, initialization of the EKF may not be as straightforward as in a desktop simulation. In many cases, it is known how long a cell has been resting prior to battery demand by the application, so initializing the dynamic states to zero may be reasonable. However, state of charge must often be inferred from an initial cell voltage measurement, which can introduce error. So, in this section we investigate how quickly the EKF can recover from an incorrect initial state of charge (modeled by changing the $c_{s, 0}$ constant in both electrodes to an incorrect value). The true initial state of charge was again $60 \%$; however, the EKF was initialized to believe that the cell SOC was $40 \%$. This is an extreme example. We would expect much better initialization than this in practice, but sought to stress the filter to see how well it would perform.

The EKF estimates, in theory, should converge to the neighborhood of the truth fairly quickly, as the filter will continually try to reduce the output error by appropriately changing the values of the Kalman gain. The estimates for SOC, as depicted in Fig. 9 converge to the neighborhood of the truth values in approximately $15 \mathrm{~s}$. (The simulations were run for a full $1500 \mathrm{~s}$, but only the first $120 \mathrm{~s}$ are shown in the plots in this section as convergence occurred far more quickly than that.) The SOC-estimate bounds always encompass the truth, so we conclude that the EKF produces reliable estimates and bounds, and is robust to initialization errors.

[Figure 9 about here.] 
Estimation results for internal electrochemical variables $j_{k}(x), \phi_{s, k}(x), \phi_{e, k}(x), c_{s, e, k}(x)$, and $c_{e, k}(x)$ are presented in Figs. 10 through 14, respectively. From these plots, we see that only $\phi_{s, k}^{\mathrm{pos}}(x), \phi_{e, k}(x)$, and $c_{s, e, k}(x)$ are affected by the incorrect initialization. Convergence toward the results seen in Section 5.1 also occur within about $15 \mathrm{~s}$. In all other respects, these estimates of cell internal electrochemical variables resemble those seen in Section 5.1, including how frequently the error bounds are incorrect.

[Figure 10 about here.]

[Figure 11 about here.]

[Figure 12 about here.]

[Figure 13 about here.]

[Figure 14 about here.]

\section{Summary}

In this paper we have presented an application of the EKF to estimate the internal, electrochemical state variables of a lithium-ion battery cell. We have utilized in the EFK a computationally efficient, low-order, discrete-time statespace model reduced from a full order mathematical description derived from first principles. To our knowledge, this is the first successful application of an EKF for internal cell state estimation that delivers confidence bounds on the estimated quantities. Results show excellent performance in simulation where internal variables estimated from a fifth-order discrete-time model using current, voltage and temperature as the only measurable quantities, matched closely with "truth" data computed from a full-order multi-physics simulation.

The use of an extended Kalman filter combined with our novel modeling methodology enables highly accurate predictions of otherwise unmeasurable electrochemical quantities. It is viewed that this approach will enable advanced predictive control methods for lithium-ion cells that can exploit the electrochemical state in order to impose practical limits in order to extend life and improve performance.

\section{Acknowledgement}

The information, data, or work presented herein was funded in part by the General Motors/University of Michigan Advanced Battery Coalition for Drivetrains (GM/UM ABCD), to which the University of Colorado Colorado Springs is a subcontractor, and by the Advanced Research Projects Agency-Energy (ARPA-E), U.S. Department of Energy, under Award Number DE-AR0000271, and by the Department of National Energy Technology Lab under Award Number DE-EE0005580.

Further, this work used the EAS Data Center or EAS Cloud, which is supported by the College of Engineering and Applied Science, University of Colorado Colorado Springs.

The authors would like to express their gratitude to each of these sponsors. 
Disclaimer. The information, data, or work presented herein was funded in part by an agency of the United States Government. Neither the United States Government nor any agency thereof, nor any of their employees, makes any warranty, express or implied, or assumes any legal liability or responsibility for the accuracy, completeness, or usefulness of any information, apparatus, product, or process disclosed, or represented that its use would not infringe privately owned rights. Referenced herein to any specific commercial product, process, or service by trade name, trademark, manufacturer, or otherwise does not necessarily constitute or imply its endorsement, recommendation, or favoring by the United States Government or any agency thereof. The views and opinions of authors expresses herein do not necessarily state or reflect those of the United States Government or any agency thereof.

\section{References}

\section{References}

[1] S. Piller, M. Perrin, A. Jossen, Journal of Power Sources 96 (2001) 113-120.

[2] S. J. Julier, J. K. Uhlmann, in: Proc. AeroSense: 11th Int. Symp. Aerospace/Defense Sensing, Simulation and Controls, volume 3068, pp. 182-193.

[3] X. Hu, S. Li, H. Peng, Journal of Power Sources 198 (2012) 359-367.

[4] G. L. Plett, Journal of Power Sources 134 (2004) 252-261.

[5] G. L. Plett, Journal of Power Sources 134 (2004) 262-276.

[6] G. L. Plett, Journal of Power Sources 134 (2004) 277-292.

[7] M. Doyle, T. F. Fuller, J. Newman, Journal of the Electrochemical Society 140 (1993) 1526-1533.

[8] T. F. Fuller, M. Doyle, J. Newman, Journal of the Electrochemical Society 141 (1994) 1-10.

[9] K. A. Smith, Electrochemical Modeling, Estimation and Control of Lithium Ion Batteries, Ph.D. thesis, Pennsylvania State University, 2006.

[10] K. A. Smith, C. D. Rahn, C.-Y. Wang, IEEE Transactions on Control Systems Technology 18 (2010) 654-663.

[11] K. A. Smith, C.-Y. Wang, Journal of Power Sources 160 (2006) 662-673.

[12] J. Maciejowski, Predictive Control with Constraints, Prentice Hall: Harlow, England, 2002.

[13] S. Santhanagopalan, R. E. White, Journal of Power Sources 161 (2006) 1346-1355.

[14] S. Santhanagopalan, R. White, in: Control Applications, 2008. CCA 2008. IEEE International Conference on, pp. 690-695. 
[15] D. Di Domenico, G. Fiengo, A. Stefanopoulou, in: Control Applications, 2008. CCA 2008. IEEE International Conference on, pp. 702-707.

[16] D. D. Domenico, A. Stefanopoulou, G. Fiengo, Journal of Dynamic Systems, Measurement, and Control 132 (2010) 061302.

[17] R. Klein, N. Chaturvedi, J. Christensen, J. Ahmed, R. Findeisen, A. Kojic, in: American Control Conference (ACC), 2010, pp. 6618-6623.

[18] R. Klein, N. Chaturvedi, J. Christensen, J. Ahmed, R. Findeisen, A. Kojic, Control Systems Technology, IEEE Transactions on 21 (2013) 289-301.

[19] K. Smith, C. Rahn, C.-Y. Wang, in: Control Applications, 2008. CCA 2008. IEEE International Conference on, pp. 714-719.

[20] J. L. Lee, A. Chemistruck, G. L. Plett, Journal of Power Sources 206 (2012) 367-377.

[21] J. L. Lee, A. Chemistruck, G. L. Plett, Journal of Power Sources 220 (2012) 430-448.

[22] J. L. Lee, L. L. Aldrich, K. D. Stetzel, G. L. Plett, Journal of Power Sources 255 (2014) 85-100.

[23] M. Doyle, J. Newman, A. S. Gozdz, C. N. Schmutz, J.-M. Tarascon, Journal of the Electrochemical Society 143 (1996) 1890-1903.

[24] N. J. Higham, Linear Algebra and its Applications 103 (1988) 103-118.

\section{Nomenclature}

A current-collector plate area of the electrode, $\mathrm{m}^{2}$

A state transition matrix of the state-space model

$\hat{\mathbf{A}}_{k}^{+} \quad$ linearization of state equation evaluated using state estimate $\hat{\mathbf{x}}_{k}^{+}$

B input matrix of the state-space model

$\hat{\mathbf{B}}_{k} \quad$ linearization of state equation evaluated using state estimate $\hat{\mathbf{x}}_{k}^{+}$

C output matrix of the state-space model

$\hat{\mathbf{C}}_{k}^{-} \quad$ linearization of output function evaluated using state prediction $\hat{\mathbf{x}}_{k}^{-}$

$\hat{\mathbf{C}}_{k}^{+} \quad$ linearization of internal-variables function evaluated using state estimate $\hat{\mathbf{x}}_{k}^{+}$

c concentration of lithium in phase indicated by subscript, $\mathrm{mol} \mathrm{m}^{-3}$

$c_{e, 0} \quad$ steady-state concentration of lithium in the electrolyte phase, $\mathrm{mol} \mathrm{m}^{-3}$ 


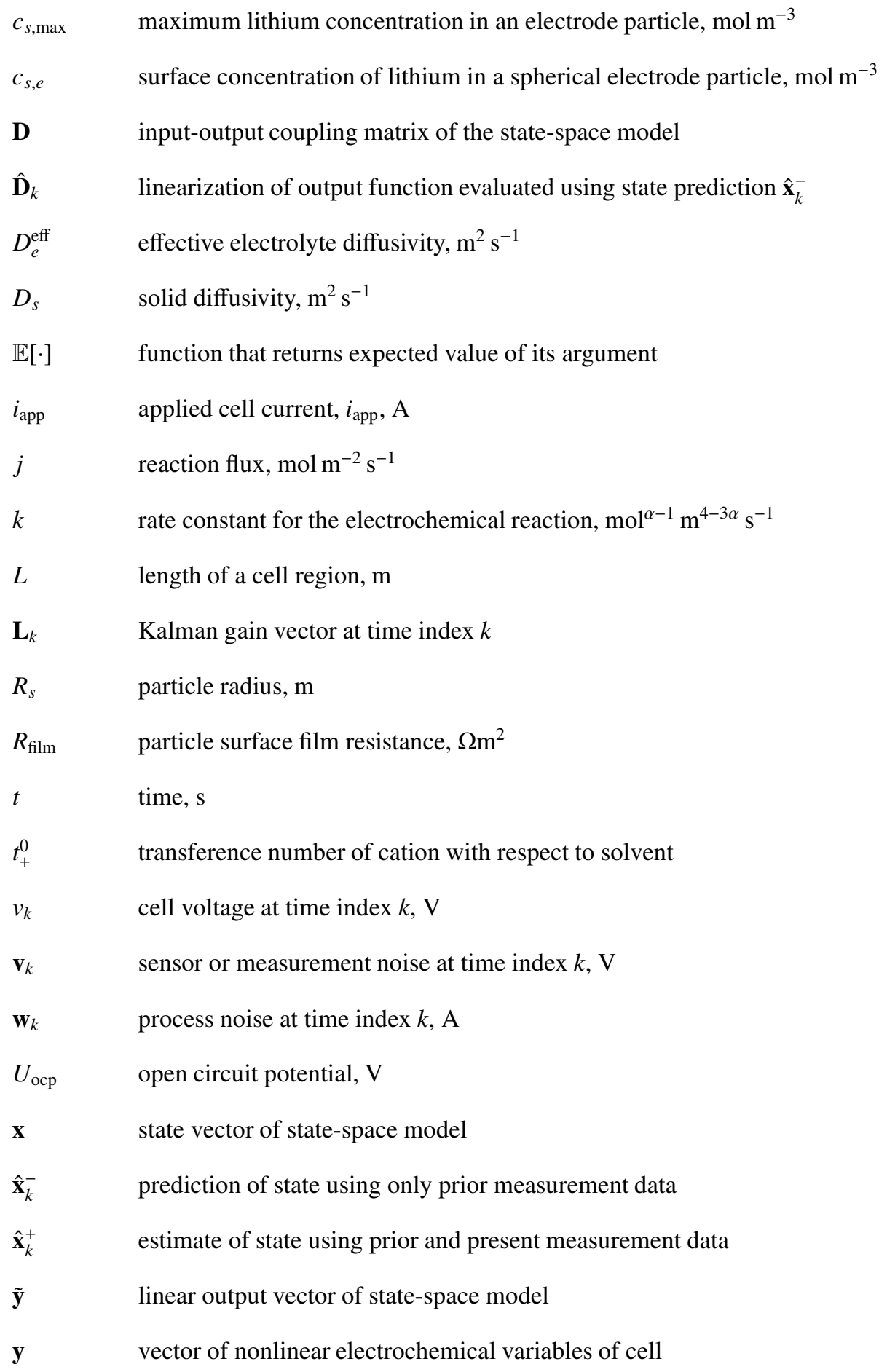

Greek

$\alpha \quad$ charge-transfer coefficient 


$\begin{array}{ll}\eta & \text { overpotential, } \mathrm{V} \\ \phi & \text { potential of the phase indicated by subscript, } \mathrm{V} \\ \kappa^{\mathrm{eff}} & \text { effective electrolyte conductivity, } \mathrm{S} \mathrm{m}^{-2} \\ \sigma^{\mathrm{eff}} & \text { effective solid conductivity, } \mathrm{S} \mathrm{m}^{-2} \\ \theta & \text { electrode stoichiometry } \\ \Sigma & \text { covariance matrix of quantity indicated in subscript }\end{array}$

\section{Subscript/superscript}

$\begin{array}{ll}e & \text { pertaining to the electrolyte phase } \\ k & \text { discrete-time sample index } \\ \text { neg } & \text { pertaining to the negative electrode } \\ \text { pos } & \text { pertaining to the positive electrode } \\ s & \text { pertaining to the solid phase }\end{array}$




\section{List of Figures}

1 Pseudo-two-dimensional porous-electrode model. . . . . . . . . . . . . . . . 23

2 Input current profile. . . . . . . . . . . . . . . . . . . . . . . 24

3 Comparing SOC estimate of EKF to truth: (a) FOM vs. EKF; (b) estimation error . . . . . . . . . . . 25

4 Comparing flux estimate of EKF to truth: (a) and (c) FOM vs. EKF; (b) and (d) estimation error . . . 26

5 Comparing $\phi_{s}$ estimate of EKF to truth: (a) and (c) FOM vs. EKF; (b) and (d) estimation error . . . . 27

6 Comparing $\phi_{e}$ estimate of EKF to truth: (a) and (c) FOM vs. EKF; (b) and (d) estimation error . . . . 28

$7 \quad$ Comparing $c_{s, e}$ estimate of EKF to truth: (a) and (c) FOM vs. EKF; (b) and (d) estimation error . . . . 29

8 Comparing $c_{e}$ estimate of EKF to truth: (a) and (c) FOM vs. EKF; (b) and (d) estimation error . . . . 30

9 Comparing SOC estimate of EKF to truth: (a) FOM vs. EKF; (b) estimation error . . . . . . . . . . . 31

10 Comparing flux estimate of EKF to truth: (a) and (c) FOM vs. EKF; (b) and (d) estimation error . . . 32

11 Comparing $\phi_{s}$ estimate of EKF to truth: (a) and (c) FOM vs. EKF; (b) and (d) estimation error . . . . 33

12 Comparing $\phi_{e}$ estimate of EKF to truth: (a) and (c) FOM vs. EKF; (b) and (d) estimation error . . . . 34

13 Comparing $c_{s, e}$ estimate of EKF to truth: (a) and (c) FOM vs. EKF; (b) and (d) estimation error . . . . 35

14 Comparing $c_{e}$ estimate of EKF to truth: (a) and (c) FOM vs. EKF; (b) and (d) estimation error . . . . 36 


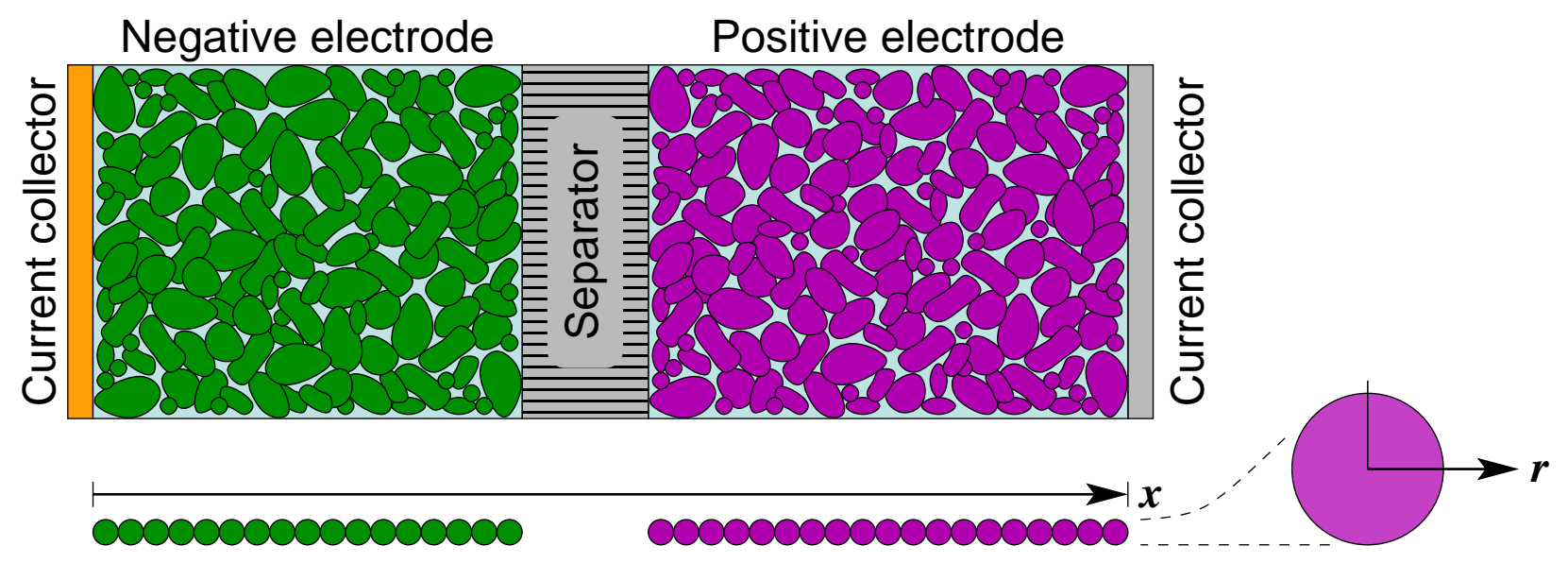

Figure 1: Pseudo-two-dimensional porous-electrode model. 


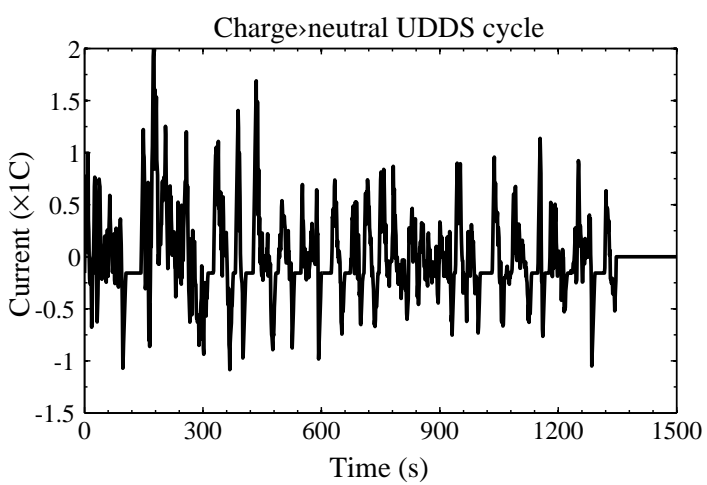

Figure 2: Input current profile. 

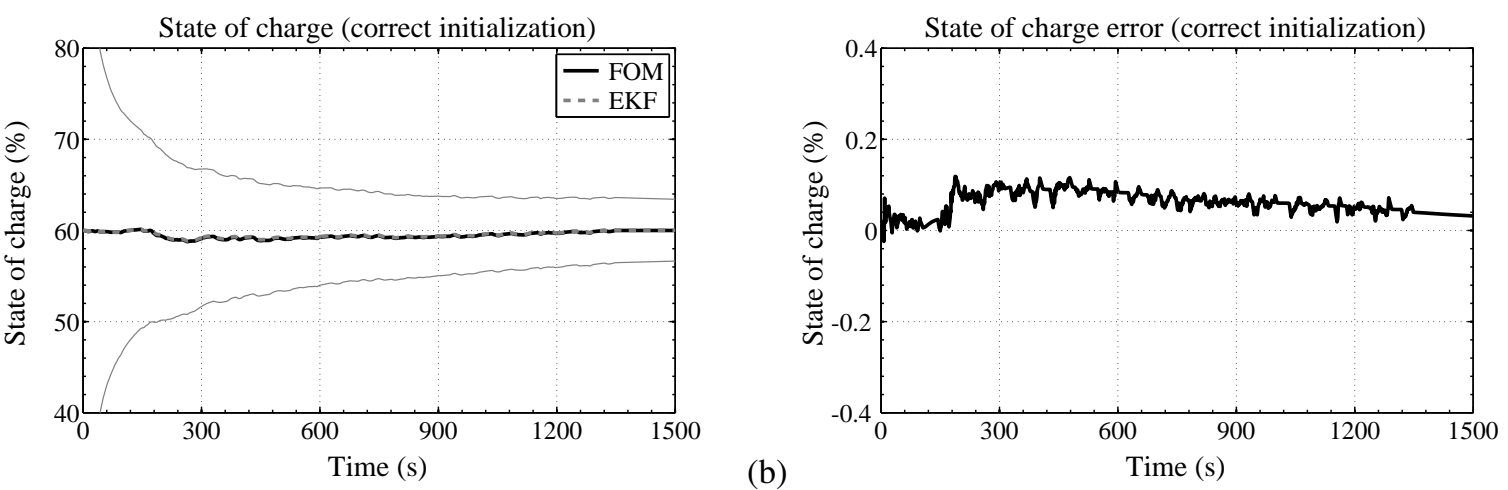

Figure 3: Comparing SOC estimate of EKF to truth: (a) FOM vs. EKF; (b) estimation error 

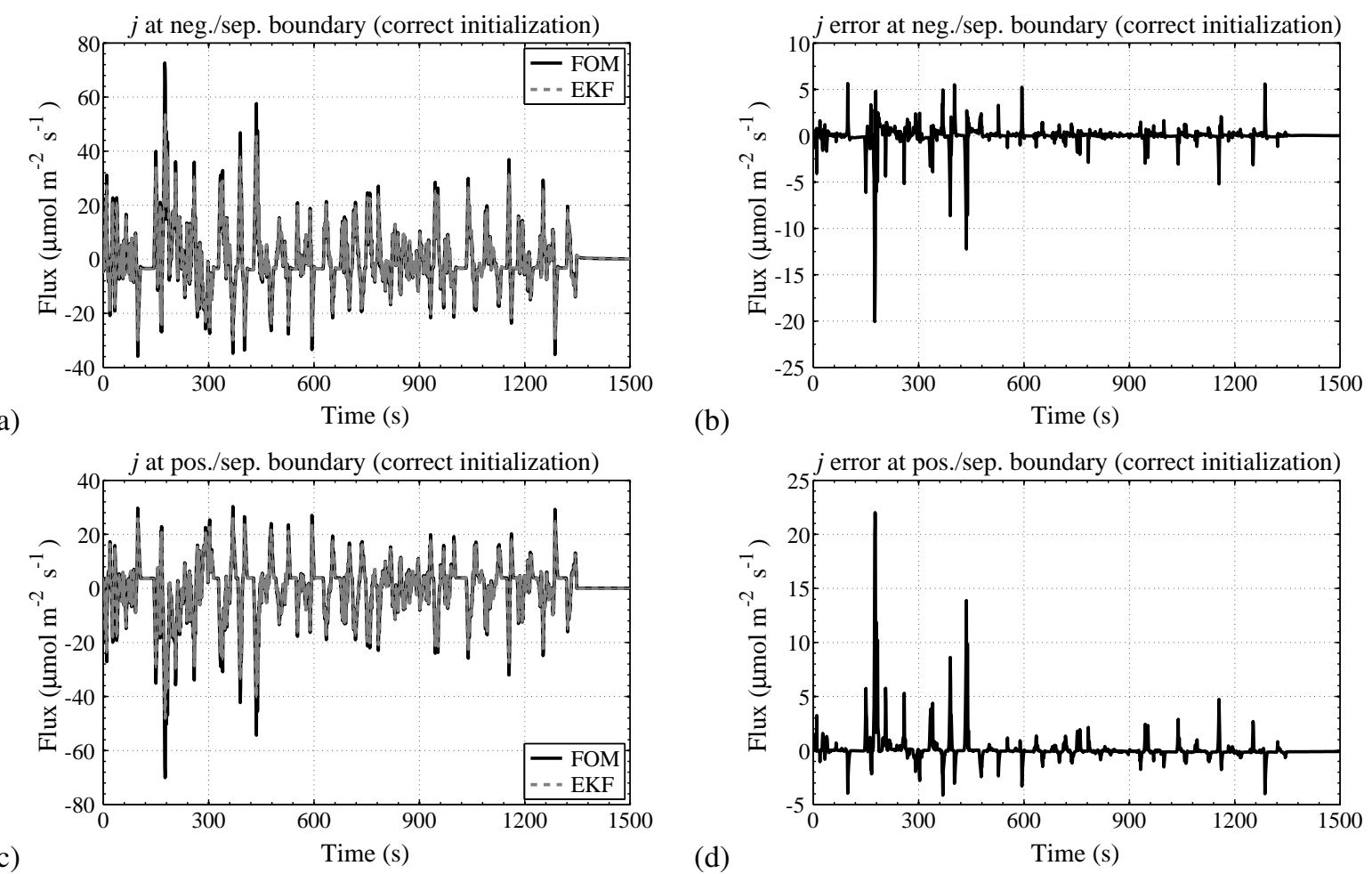

Figure 4: Comparing flux estimate of EKF to truth: (a) and (c) FOM vs. EKF; (b) and (d) estimation error 

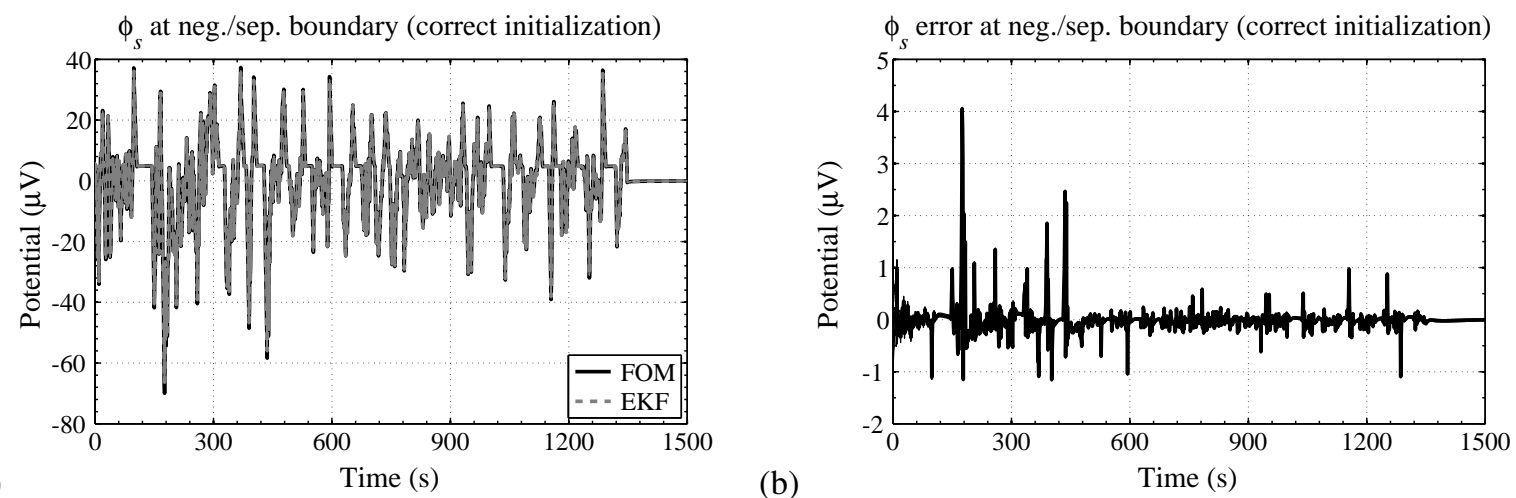

(a)

(b)
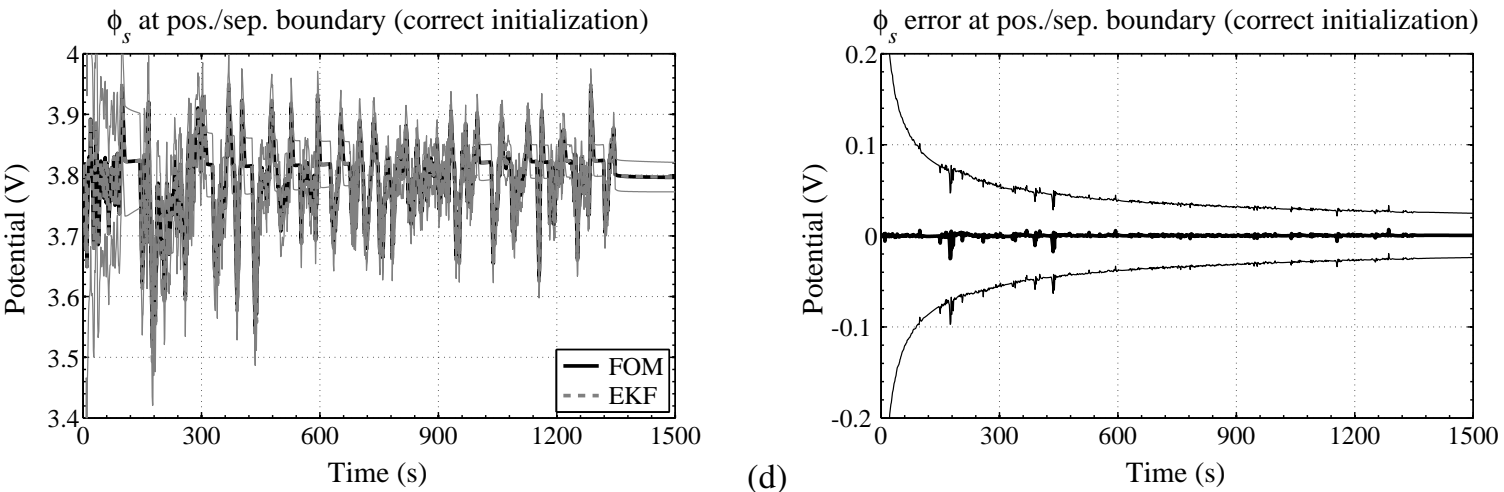

Figure 5: Comparing $\phi_{s}$ estimate of EKF to truth: (a) and (c) FOM vs. EKF; (b) and (d) estimation error 


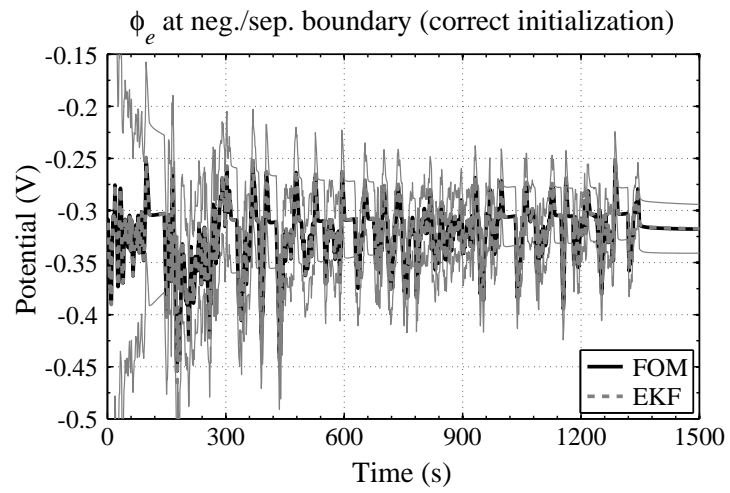

(a)

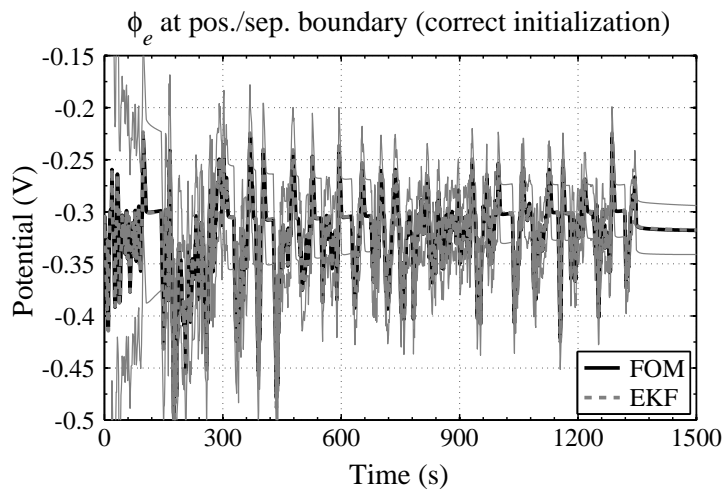

(b)
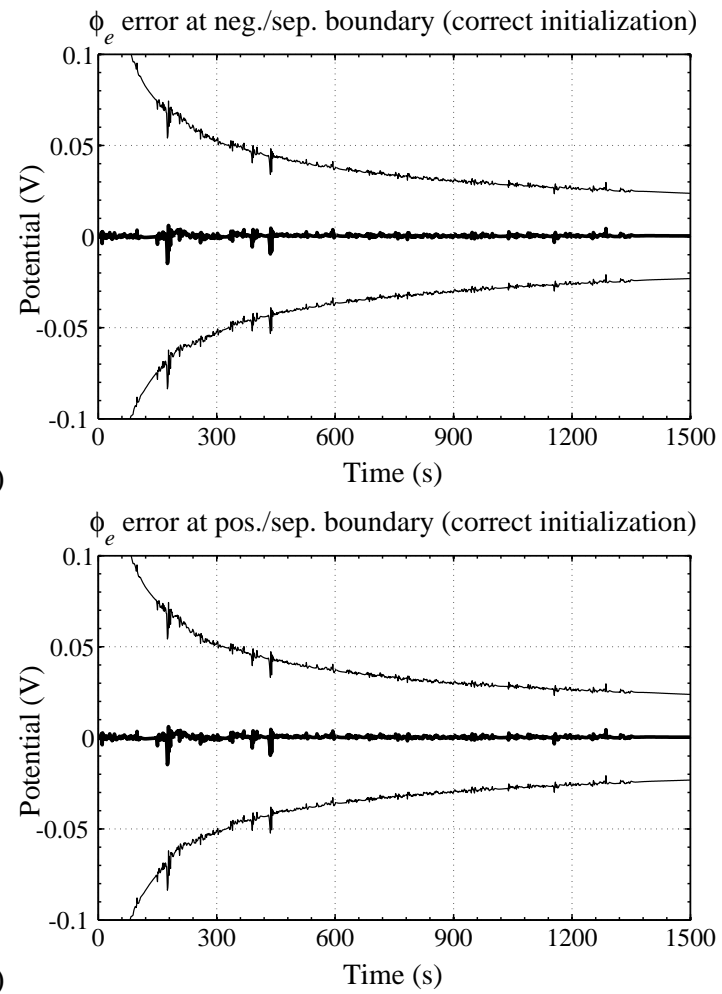

Figure 6: Comparing $\phi_{e}$ estimate of EKF to truth: (a) and (c) FOM vs. EKF; (b) and (d) estimation error 


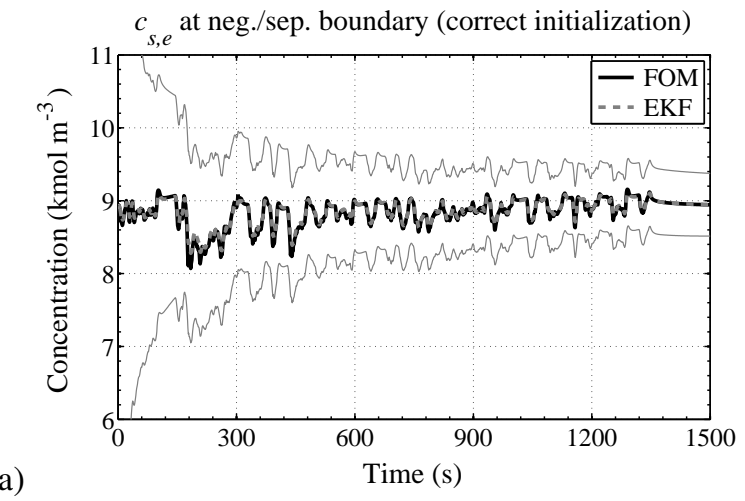

(a)

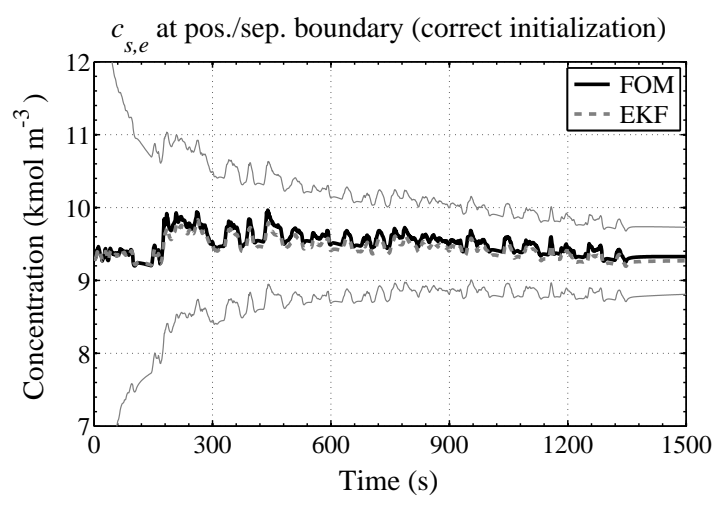

(b)

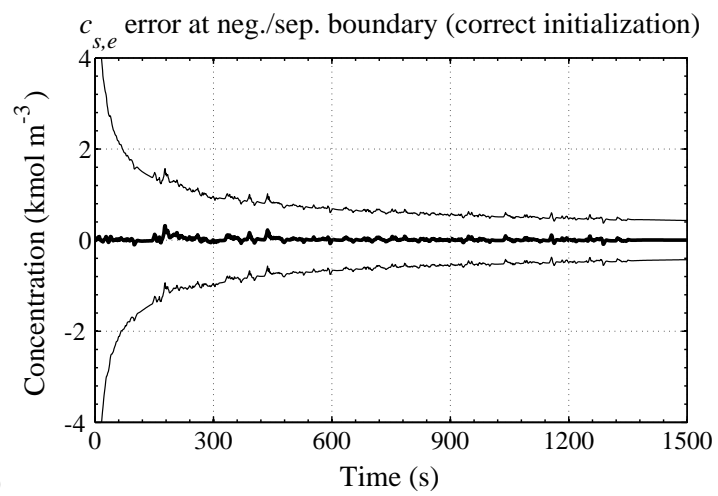

(d)

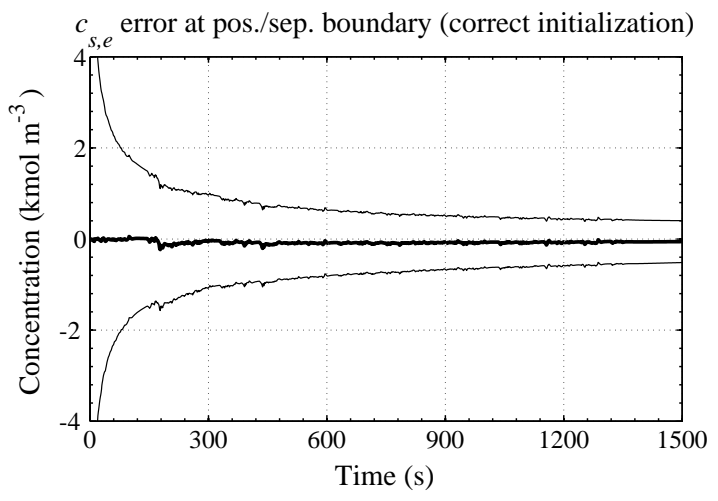

Figure 7: Comparing $c_{s, e}$ estimate of EKF to truth: (a) and (c) FOM vs. EKF; (b) and (d) estimation error 

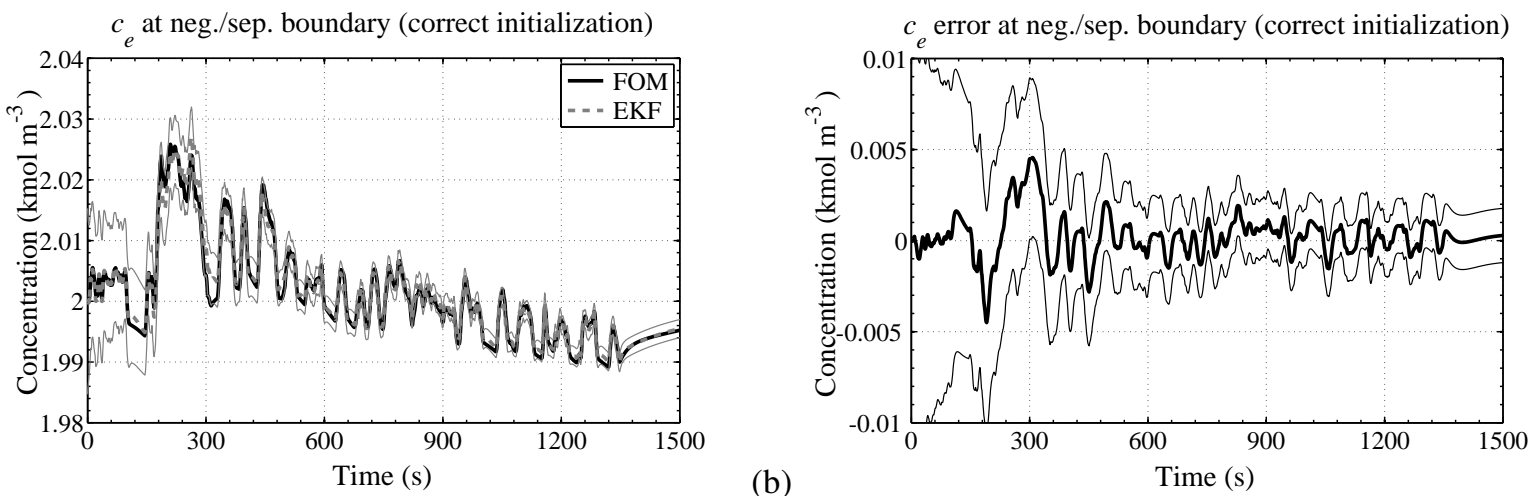

(a)

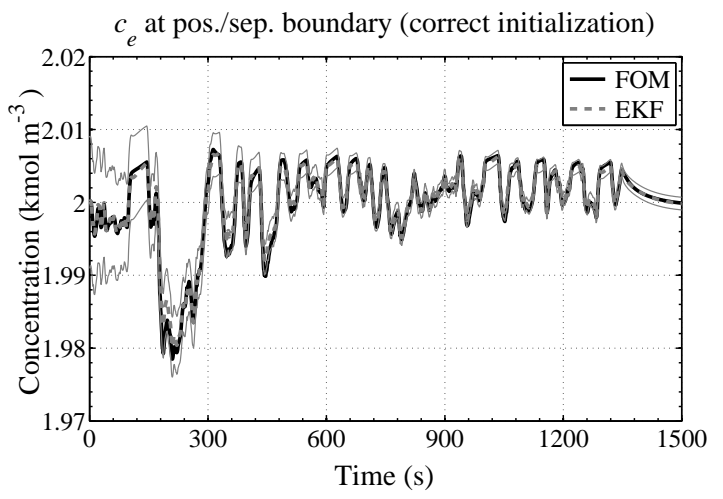

(b)

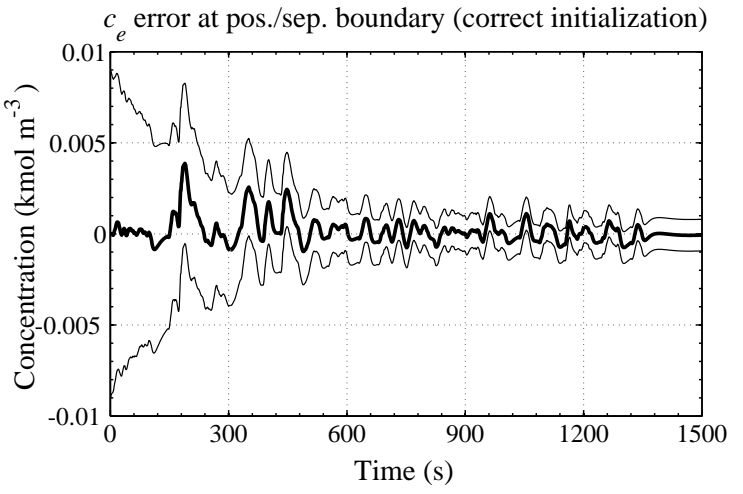

Figure 8: Comparing $c_{e}$ estimate of EKF to truth: (a) and (c) FOM vs. EKF; (b) and (d) estimation error 

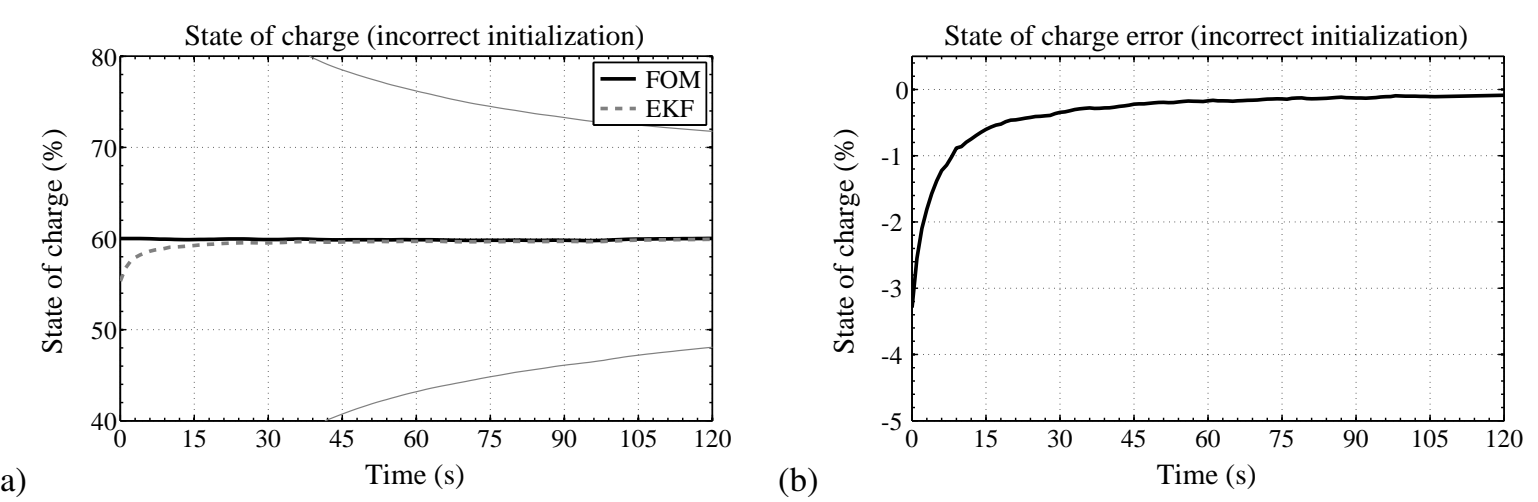

Figure 9: Comparing SOC estimate of EKF to truth: (a) FOM vs. EKF; (b) estimation error 

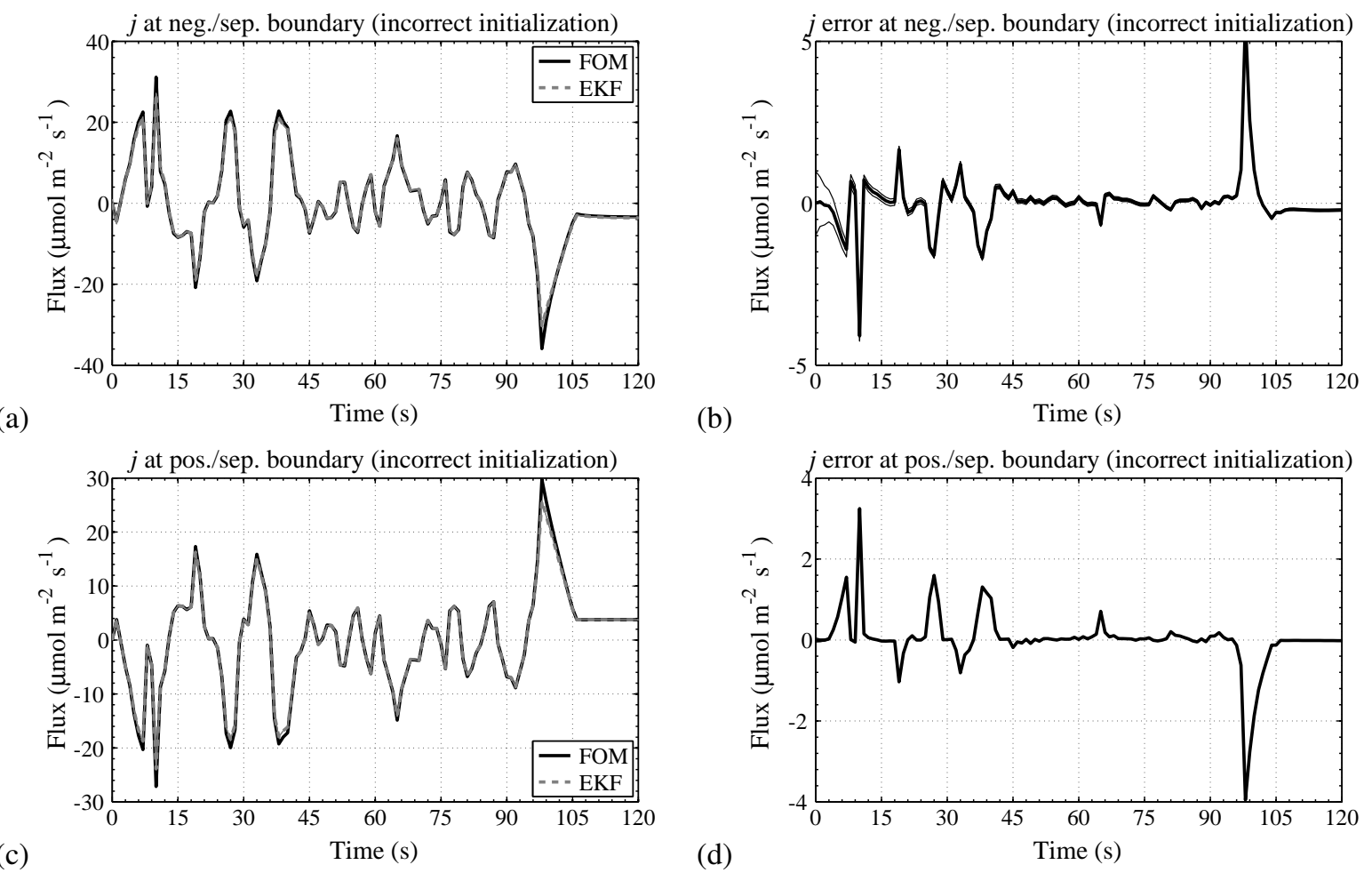

Figure 10: Comparing flux estimate of EKF to truth: (a) and (c) FOM vs. EKF; (b) and (d) estimation error 


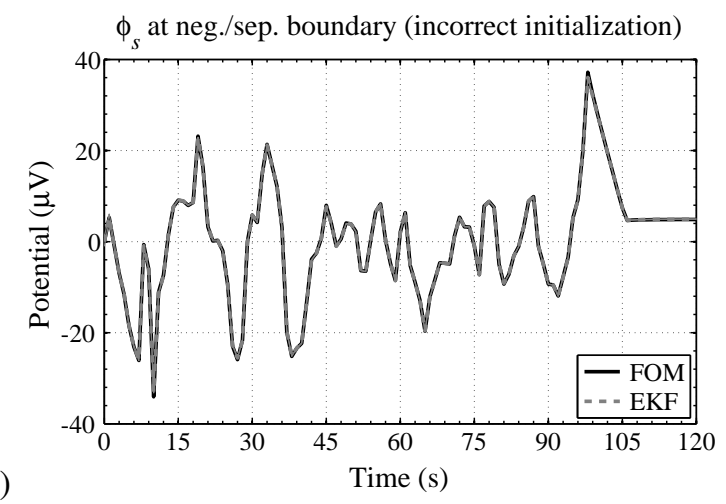

(b)
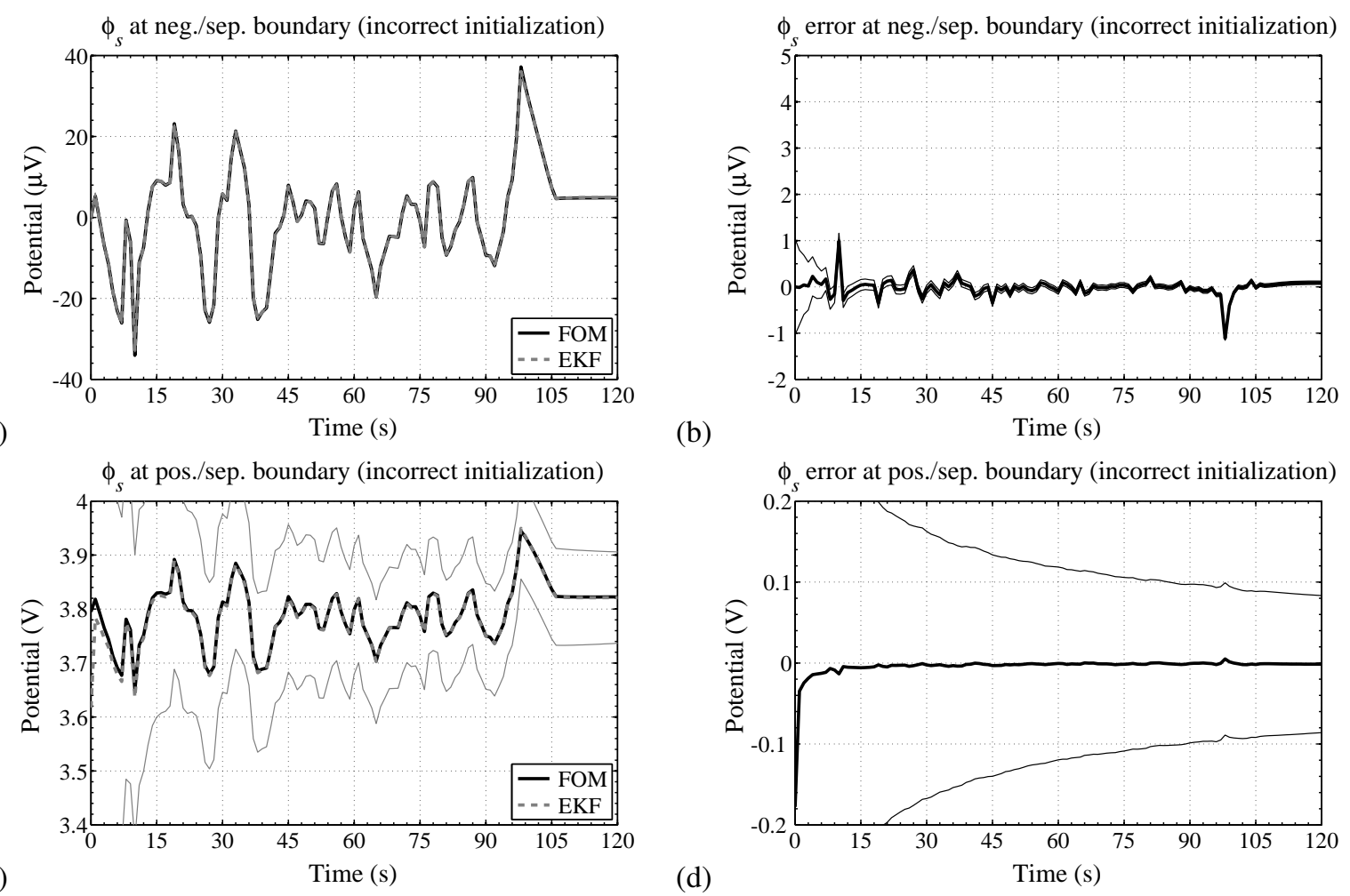

Figure 11: Comparing $\phi_{s}$ estimate of EKF to truth: (a) and (c) FOM vs. EKF; (b) and (d) estimation error 


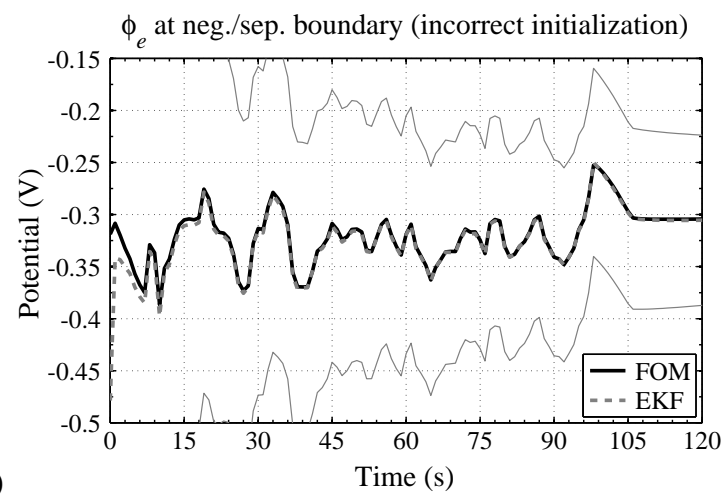

(a)
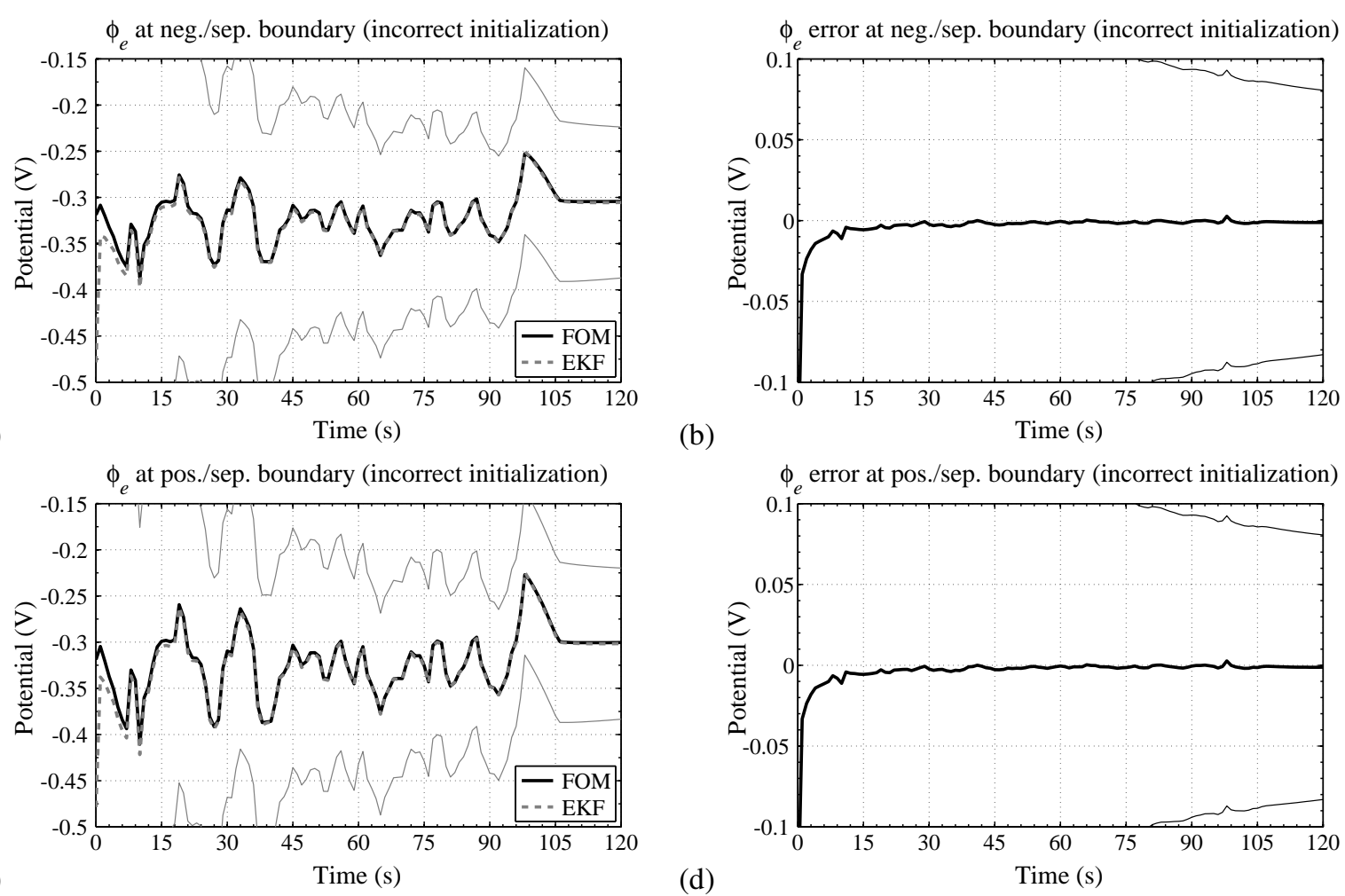

(b)

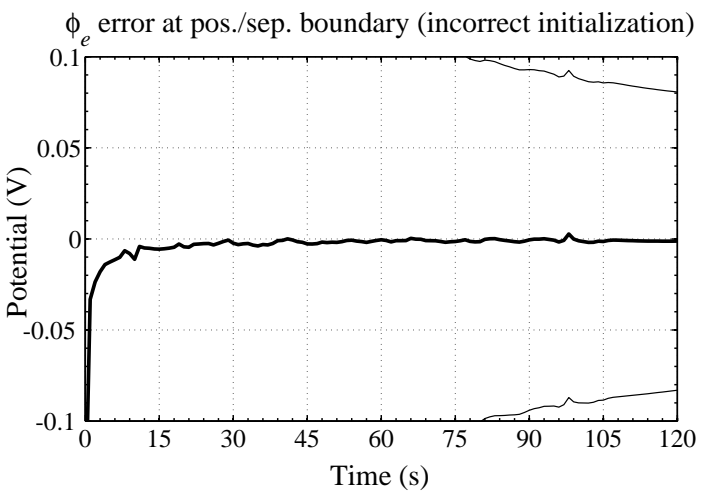

Figure 12: Comparing $\phi_{e}$ estimate of EKF to truth: (a) and (c) FOM vs. EKF; (b) and (d) estimation error 


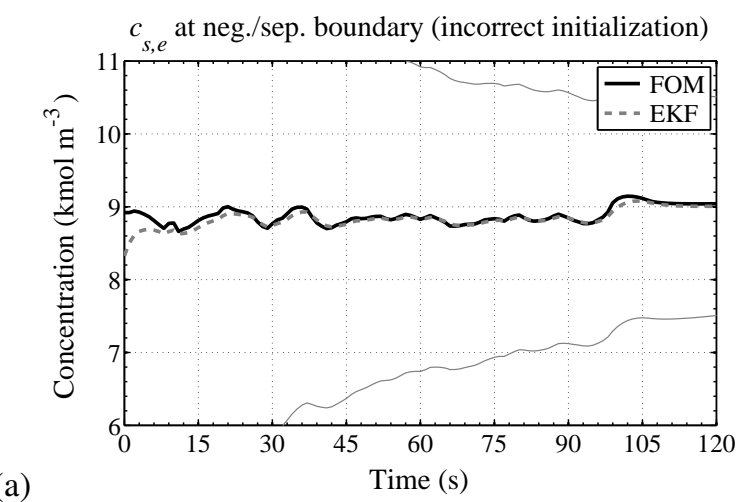

(a)

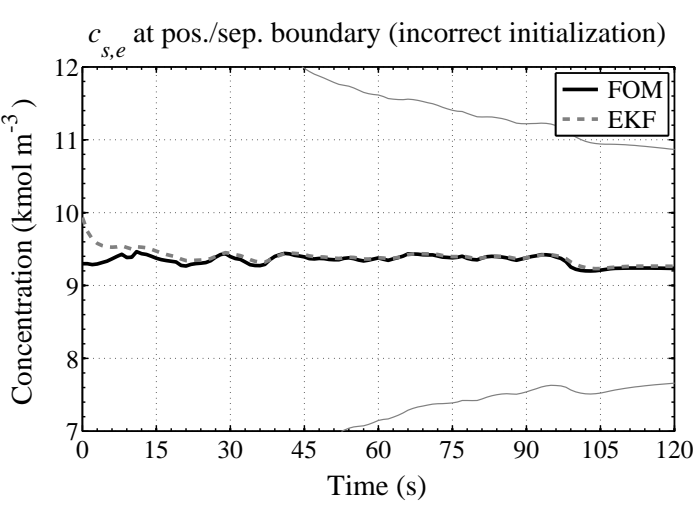

(b)
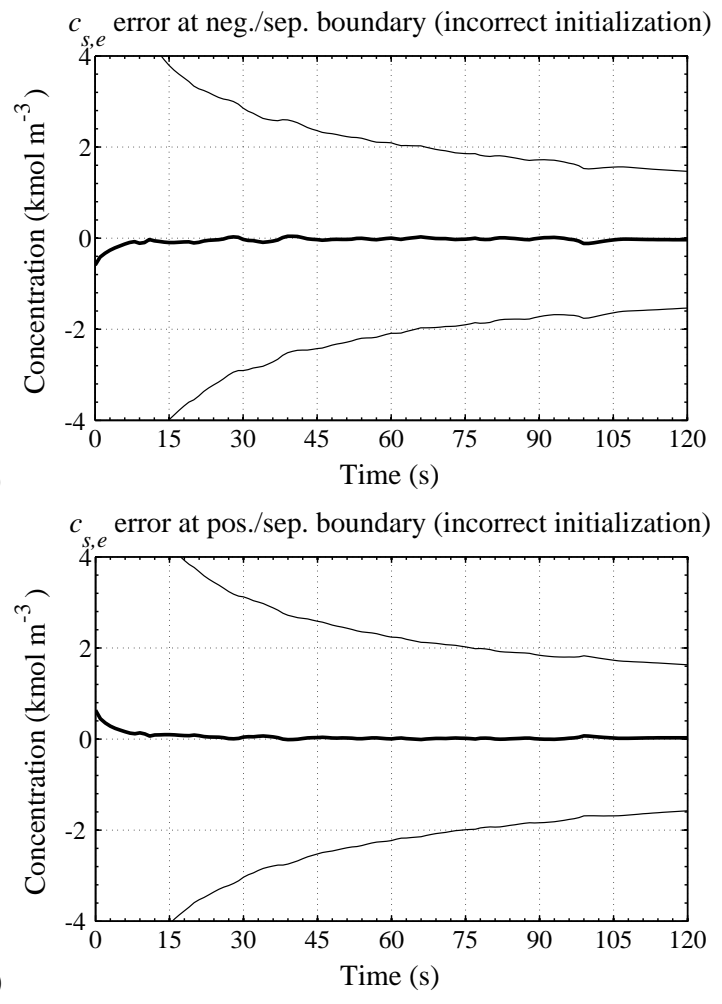

(d)

Figure 13: Comparing $c_{s, e}$ estimate of EKF to truth: (a) and (c) FOM vs. EKF; (b) and (d) estimation error 


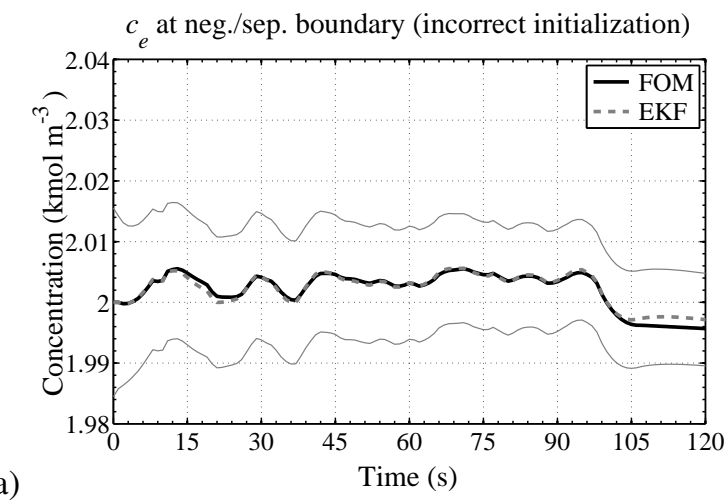

(a)

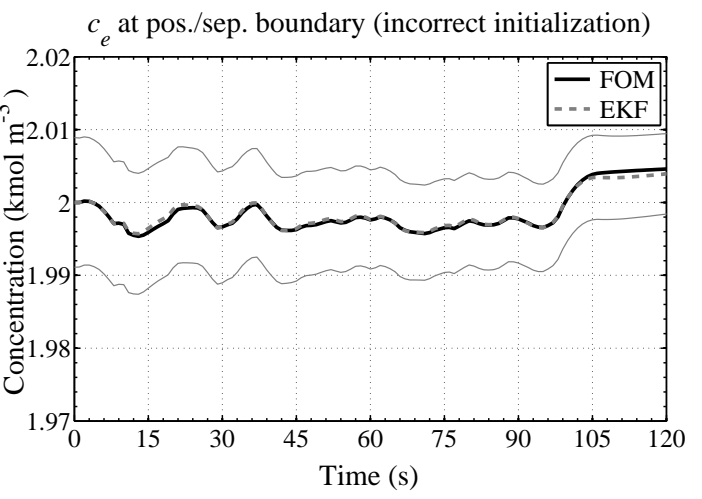

(b)
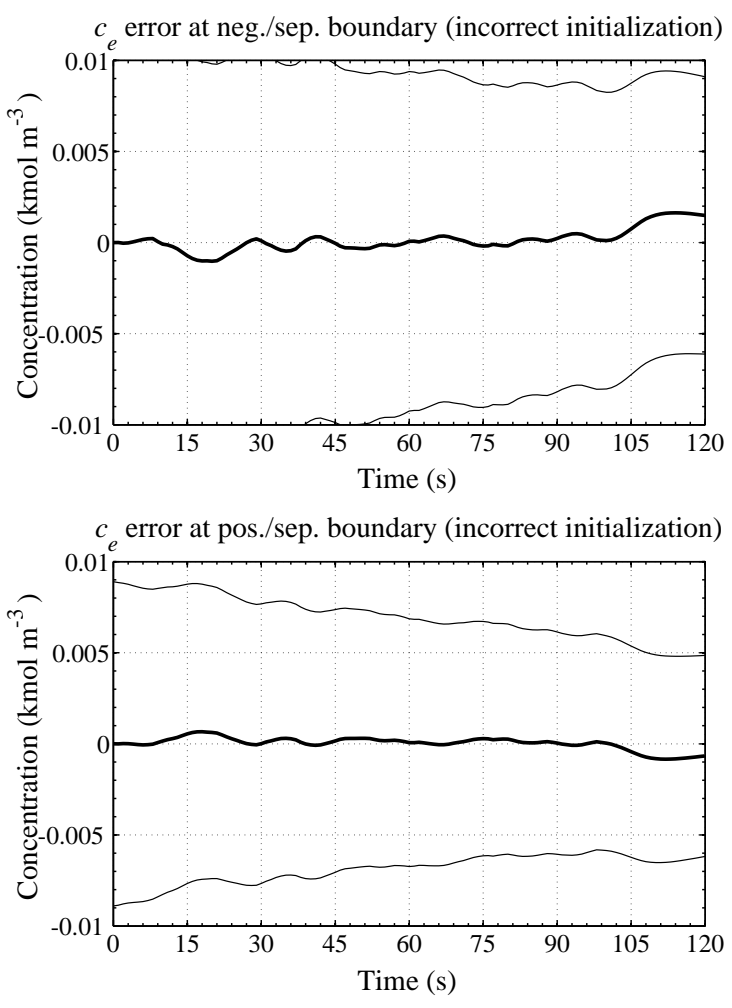

(d)

Figure 14: Comparing $c_{e}$ estimate of EKF to truth: (a) and (c) FOM vs. EKF; (b) and (d) estimation error 


\section{List of Tables}

1 Summary of the nonlinear extended Kalman filter, including internal variables estimation. . . . . . . 38

2 Cell parameters for simulation. . . . . . . . . . . . . . . . . . . . . . 39 


\section{Nonlinear state-space model:}

$$
\begin{array}{rlrl}
\mathbf{x}_{k+1} & =f_{k}\left(\mathbf{x}_{k}, \mathbf{u}_{k}, \mathbf{w}_{k}\right) & & \ldots \text { process equation } \\
\mathbf{y}_{k} & =g_{k}\left(\mathbf{x}_{k}, \mathbf{u}_{k}\right) & \ldots \text { internal variables calculation } \\
\mathbf{z}_{k} & =h_{k}\left(\mathbf{x}_{k}, \mathbf{u}_{k}, \mathbf{v}_{k}\right) & \ldots \text { measurement equation, }
\end{array}
$$

where $\mathbf{w}_{k}$ and $\mathbf{v}_{k}$ are independent Gaussian noise processes with means $\overline{\mathbf{w}}_{k}$ and $\overline{\mathbf{v}}_{k}$, respectively, and having covariance matrices $\Sigma_{\widetilde{\mathbf{w}}}$ and $\Sigma_{\tilde{\mathbf{v}}}$, respectively.

\section{Definitions:}

$$
\begin{array}{ll}
\hat{\mathbf{A}}_{k}^{+}=\left.\frac{\mathrm{d} f_{k}\left(\mathbf{x}_{k}, \mathbf{u}_{k}, \mathbf{w}_{k}\right)}{\mathrm{d} \mathbf{x}_{k}}\right|_{\mathbf{x}_{k}=\hat{\mathbf{x}}_{k}^{+}} & \hat{\mathbf{B}}_{k}=\left.\frac{\mathrm{d} f_{k}\left(\mathbf{x}_{k}, \mathbf{u}_{k}, \mathbf{w}_{k}\right)}{\mathrm{d} \mathbf{w}_{k}}\right|_{\mathbf{w}_{k}=\overline{\mathbf{w}}_{k}} \\
\hat{\mathbf{C}}_{k}^{-}=\left.\frac{\mathrm{d} h_{k}\left(\mathbf{x}_{k}, \mathbf{u}_{k}, \mathbf{v}_{k}\right)}{\mathrm{d} \mathbf{x}_{k}}\right|_{\mathbf{x}_{k}=\hat{\mathbf{x}}_{k}^{-}} & \hat{\mathbf{D}}_{k}=\left.\frac{\mathrm{d} h_{k}\left(\mathbf{x}_{k}, \mathbf{u}_{k}, \mathbf{v}_{k}\right)}{\mathrm{d} \mathbf{v}_{k}}\right|_{\mathbf{v}_{k}=\overline{\mathbf{v}}_{k}} \\
\hat{\mathbf{C}}_{k}^{+}=\left.\frac{\mathrm{d} g_{k}\left(\mathbf{x}_{k}, \mathbf{u}_{k}\right)}{\mathrm{d} \mathbf{x}_{k}}\right|_{\mathbf{x}_{k}=\hat{\mathbf{x}}_{k}^{+}} &
\end{array}
$$

Initialization: For $k=0$, set

$$
\begin{aligned}
\hat{\mathbf{x}}_{0}^{+} & =\mathbb{E}\left[\mathbf{x}_{0}\right] \\
\Sigma_{\tilde{\mathbf{x}}, 0}^{+} & =\mathbb{E}\left[\left(\mathbf{x}_{0}-\hat{\mathbf{x}}_{0}^{+}\right)\left(\mathbf{x}_{0}-\hat{\mathbf{x}}_{0}^{+}\right)^{T}\right] .
\end{aligned}
$$

Computation: For $k=1,2, \ldots$ compute:

Step 1: Prediction based on propagating prior estimate

1a. State prediction time update:

$$
\hat{\mathbf{x}}_{k}^{-}=f_{k-1}\left(\hat{\mathbf{x}}_{k-1}^{+}, \mathbf{u}_{k-1}, \overline{\mathbf{w}}_{k-1}\right) \text {. }
$$

1b. Error covariance time update:

$$
\Sigma_{\tilde{\mathbf{x}}, k}^{-}=\left(\hat{\mathbf{A}}_{k-1}^{+}\right) \Sigma_{\tilde{\mathbf{x}}, k-1}^{+}\left(\hat{\mathbf{A}}_{k-1}^{+}\right)^{T}+\hat{\mathbf{B}}_{k-1} \Sigma_{\widetilde{\mathbf{w}}} \hat{\mathbf{B}}_{k-1}^{T} .
$$

1c. Output prediction:

$$
\hat{\mathbf{z}}_{k}=h_{k}\left(\hat{\mathbf{x}}_{k}^{-}, \mathbf{u}_{k}, \overline{\mathbf{v}}_{k}\right) \text {. }
$$

Step 2: Measurement-based correction

2a. Estimator gain matrix:

$$
\mathbf{L}_{k}=\Sigma_{\tilde{\mathbf{x}}, k}^{-}\left(\hat{\mathbf{C}}_{k}^{-}\right)^{T}\left[\left(\hat{\mathbf{C}}_{k}^{-}\right) \Sigma_{\tilde{\mathbf{x}}, k}^{-}\left(\hat{\mathbf{C}}_{k}^{-}\right)^{T}+\hat{\mathbf{D}}_{k} \Sigma_{\tilde{\mathbf{v}}} \hat{\mathbf{D}}_{k}^{T}\right]^{-1} .
$$

2b. State estimate measurement update:

$$
\hat{\mathbf{x}}_{k}^{+}=\hat{\mathbf{x}}_{k}^{-}+\mathbf{L}_{k}\left(\mathbf{z}_{k}-\hat{\mathbf{z}}_{k}\right) \text {. }
$$

2c. Error covariance measurement update:

$$
\Sigma_{\tilde{\mathbf{x}}, k}^{+}=\Sigma_{\tilde{\mathbf{x}}, k}^{-}-\mathbf{L}_{k} \Sigma_{\tilde{\mathbf{z}}, k} \mathbf{L}_{k}^{T}
$$

Step 3: Internal variables estimate

3a. Internal electrochemical variables estimate:

$$
\hat{\mathbf{y}}_{k}=g_{k}\left(\hat{\mathbf{x}}_{k}^{+}, \mathbf{u}_{k}\right) \text {. }
$$

3b. Internal variables covariance estimate:

$$
\Sigma_{\tilde{\mathbf{y}}, k}=\left(\hat{\mathbf{C}}_{k}^{+}\right)^{T} \Sigma_{\tilde{\mathbf{x}}, k}\left(\hat{\mathbf{C}}_{k}^{+}\right) .
$$


Table 2: Cell parameters for simulation.

\begin{tabular}{ccccc}
\hline Symbol & Units & Negative electrode & Separator & Positive electrode \\
\hline$L$ & $\mu \mathrm{m}$ & 128 & 76 & 190 \\
$R_{s}$ & $\mu \mathrm{m}$ & 12.5 & - & 8.5 \\
$A$ & $\mathrm{~m}^{2}$ & 1 & 1 & 1 \\
$\sigma$ & $\mathrm{S} \mathrm{m}^{-1}$ & 100 & - & 3.8 \\
$\varepsilon_{s}$ & $\mathrm{~m}^{3} \mathrm{~m}^{-3}$ & 0.471 & - & 0.297 \\
$\varepsilon_{e}$ & $\mathrm{~m}^{3} \mathrm{~m}^{-3}$ & 0.357 & 0.724 & 0.444 \\
brug & - & 1.5 & - & 1.5 \\
$c_{s, \max }$ & $\mathrm{mol} \mathrm{m}^{-3}$ & 26390 & - & 22860 \\
$c_{e, 0}$ & $\mathrm{~mol} \mathrm{~m}^{-3}$ & 2000 & 2000 & 2000 \\
$\theta_{i, \min }$ & - & 0.05 & - & 0.78 \\
$\theta_{i, \mathrm{max}}$ & - & 0.53 & - & 0.17 \\
$D_{s}$ & $\mathrm{~m}^{2} \mathrm{~s}^{-1}$ & $3.9 \times 10^{-14}$ & - & $1.0 \times 10^{-13}$ \\
$D_{e}$ & $\mathrm{~m}^{2} \mathrm{~s}^{-1}$ & $7.5 \times 10^{-11}$ & $7.5 \times 10^{-11}$ & $7.5 \times 10^{-11}$ \\
$t_{+}^{0}$ & - & 0.363 & 0.363 & 0.363 \\
$k$ & $\mathrm{~mol}^{-1 / 2} \mathrm{~m}^{5 / 2} \mathrm{~s}^{-1}$ & $1.94 \times 10^{-11}$ & - & $2.16 \times 10^{-11}$ \\
$\alpha$ & - & 0.5 & - & 0.5 \\
$R_{\mathrm{film}}$ & $\Omega^{2}$ & 0.0 & - & -
\end{tabular}

We compute $\sigma^{\text {eff }}=\sigma \varepsilon_{s}, \kappa^{\text {eff }}=\kappa \varepsilon_{e}^{\text {brug }}, D_{e}^{\text {eff }}=D_{e} \varepsilon_{e}^{\text {brug }}$.

In the electrolyte, conductivity is a function of concentration:

$$
\begin{gathered}
\kappa\left(c_{e}\right)=4.1253 \times 10^{-2}+5.007 \times 10^{-4} c_{e}-4.7212 \times 10^{-7} c_{e}^{2} \\
+1.5094 \times 10^{-10} c_{e}^{3}-1.6018 \times 10^{-14} c_{e}^{4} .
\end{gathered}
$$

For the negative electrode, the open-circuit potential function is:

$$
U_{\text {ocp }}(\theta)=-0.16+1.32 \exp (-3.0 \theta)+10.0 \exp (-2000.0 \theta) .
$$

For the positive electrode, the open-circuit potential function is:

$$
\begin{aligned}
& U_{\text {ocp }}(\theta)=4.19829+0.0565661 \tanh (-14.5546 \theta+8.60942) \\
&-0.0275479\left[\frac{1}{(0.998432-\theta)^{0.4924656}}-1.90111\right] \\
&-0.157123 \exp \left(-0.04738 \theta^{6}\right)+0.810239 \exp [-40(\theta-0.133875)] .
\end{aligned}
$$


Nonlinear state-space model:

$$
\begin{aligned}
\mathbf{x}_{k+1} & =f_{k}\left(\mathbf{x}_{k}, \mathbf{u}_{k}, \mathbf{w}_{k}\right) & \ldots \text { process equation } \\
\mathbf{y}_{k} & =g_{k}\left(\mathbf{x}_{k}, \mathbf{u}_{k}\right) & \ldots \text { internal variables calculation } \\
\mathbf{z}_{k} & =h_{k}\left(\mathbf{x}_{k}, \mathbf{u}_{k}, \mathbf{v}_{k}\right) & \ldots \text { measurement equation, }
\end{aligned}
$$

where $\mathbf{w}_{k}$ and $\mathbf{v}_{k}$ are independent Gaussian noise processes with means $\overline{\mathbf{w}}_{k}$ and $\overline{\mathbf{v}}_{k}$, respectively, and having covariance matrices $\Sigma_{\widetilde{\mathbf{w}}}$ and $\Sigma_{\tilde{\mathbf{v}}}$, respectively.

\section{Definitions:}

$$
\begin{array}{ll}
\hat{\mathbf{A}}_{k}^{+}=\left.\frac{\mathrm{d} f_{k}\left(\mathbf{x}_{k}, \mathbf{u}_{k}, \mathbf{w}_{k}\right)}{\mathrm{d} \mathbf{x}_{k}}\right|_{\mathbf{x}_{k}=\hat{\mathbf{x}}_{k}^{+}} & \hat{\mathbf{B}}_{k}=\left.\frac{\mathrm{d} f_{k}\left(\mathbf{x}_{k}, \mathbf{u}_{k}, \mathbf{w}_{k}\right)}{\mathrm{d} \mathbf{w}_{k}}\right|_{\mathbf{w}_{k}=\overline{\mathbf{w}}_{k}} \\
\hat{\mathbf{C}}_{k}^{-}=\left.\frac{\mathrm{d} h_{k}\left(\mathbf{x}_{k}, \mathbf{u}_{k}, \mathbf{v}_{k}\right)}{\mathrm{d} \mathbf{x}_{k}}\right|_{\mathbf{x}_{k}=\hat{\mathbf{x}}_{k}^{-}} & \hat{\mathbf{D}}_{k}=\left.\frac{\mathrm{d} h_{k}\left(\mathbf{x}_{k}, \mathbf{u}_{k}, \mathbf{v}_{k}\right)}{\mathrm{d} \mathbf{v}_{k}}\right|_{\mathbf{v}_{k}=\overline{\mathbf{v}}_{k}} \\
\hat{\mathbf{C}}_{k}^{+}=\left.\frac{\mathrm{d} g_{k}\left(\mathbf{x}_{k}, \mathbf{u}_{k}\right)}{\mathrm{d} \mathbf{x}_{k}}\right|_{\mathbf{x}_{k}=\hat{\mathbf{x}}_{k}^{+}} &
\end{array}
$$

Initialization: For $k=0$, set

$$
\begin{aligned}
\hat{\mathbf{x}}_{0}^{+} & =\mathbb{E}\left[\mathbf{x}_{0}\right] \\
\Sigma_{\tilde{\mathbf{x}}, 0}^{+} & =\mathbb{E}\left[\left(\mathbf{x}_{0}-\hat{\mathbf{x}}_{0}^{+}\right)\left(\mathbf{x}_{0}-\hat{\mathbf{x}}_{0}^{+}\right)^{T}\right] .
\end{aligned}
$$

Computation: For $k=1,2, \ldots$ compute:

Step 1: Prediction based on propagating prior estimate

1a. State prediction time update:

$$
\hat{\mathbf{x}}_{k}^{-}=f_{k-1}\left(\hat{\mathbf{x}}_{k-1}^{+}, \mathbf{u}_{k-1}, \overline{\mathbf{w}}_{k-1}\right) \text {. }
$$

1b. Error covariance time update:

$$
\Sigma_{\tilde{\mathbf{x}}, k}^{-}=\left(\hat{\mathbf{A}}_{k-1}^{+}\right) \Sigma_{\tilde{\mathbf{x}}, k-1}^{+}\left(\hat{\mathbf{A}}_{k-1}^{+}\right)^{T}+\hat{\mathbf{B}}_{k-1} \Sigma_{\widetilde{\mathbf{w}}} \hat{\mathbf{B}}_{k-1}^{T} .
$$

1c. Output prediction:

$$
\hat{\mathbf{z}}_{k}=h_{k}\left(\hat{\mathbf{x}}_{k}^{-}, \mathbf{u}_{k}, \overline{\mathbf{v}}_{k}\right) \text {. }
$$

Step 2: Measurement-based correction

2a. Estimator gain matrix:

$$
\mathbf{L}_{k}=\Sigma_{\tilde{\mathbf{x}}, k}^{-}\left(\hat{\mathbf{C}}_{k}^{-}\right)^{T}\left[\left(\hat{\mathbf{C}}_{k}^{-}\right) \Sigma_{\tilde{\mathbf{x}}, k}^{-}\left(\hat{\mathbf{C}}_{k}^{-}\right)^{T}+\hat{\mathbf{D}}_{k} \Sigma_{\tilde{\mathbf{v}}} \hat{\mathbf{D}}_{k}^{T}\right]^{-1} .
$$

$2 b$. State estimate measurement update:

$$
\hat{\mathbf{x}}_{k}^{+}=\hat{\mathbf{x}}_{k}^{-}+\mathbf{L}_{k}\left(\mathbf{z}_{k}-\hat{\mathbf{z}}_{k}\right) \text {. }
$$

2c. Error covariance measurement update:

$$
\Sigma_{\tilde{\mathbf{x}}, k}^{+}=\Sigma_{\tilde{\mathbf{x}}, k}^{-}-\mathbf{L}_{k} \Sigma_{\tilde{\mathbf{z}}, k} \mathbf{L}_{k}^{T} .
$$

Step 3: Internal variables estimate

3a. Internal electrochemical variables estimate:

$$
\hat{\mathbf{y}}_{k}=g_{k}\left(\hat{\mathbf{x}}_{k}^{+}, \mathbf{u}_{k}\right) \text {. }
$$

3b. Internal variables covariance estimate:

$$
\Sigma_{\tilde{\mathbf{y}}, k}=\left(\hat{\mathbf{C}}_{k}^{+}\right)^{T} \Sigma_{\tilde{\mathbf{x}}, k}\left(\hat{\mathbf{C}}_{k}^{+}\right) \text {. }
$$


Symbol

$L$

$R_{S}$

$A$

$\sigma$

$\varepsilon_{s}$

$\varepsilon_{e}$

brug

$c_{s, \max }$

$c_{e, 0}$

$\theta_{i, \min }$

$\theta_{i, \max }$
$D_{s}$
$D_{e}$
$t_{+}^{0}$
$k$
$\alpha$
$R_{\text {film }}$$$
\mathrm{m}^{2} \mathrm{~s}^{-1}
$$
$\mathrm{m}^{2} \mathrm{~s}^{-1}$

$$
\mathrm{mol}^{-1 / 2} \mathrm{~m}^{5 / 2} \mathrm{~s}^{-1}
$$
$\Omega \mathrm{m}^{2}$

Units

$\mu \mathrm{m}$

$\mu \mathrm{m}$

$\mathrm{m}^{2}$

$\mathrm{S} \mathrm{m}^{-1}$

$\mathrm{m}^{3} \mathrm{~m}^{-3}$

$\mathrm{m}^{3} \mathrm{~m}^{-3}$

$\mathrm{mol} \mathrm{m}^{-3}$

$\mathrm{mol} \mathrm{m}{ }^{-3}$

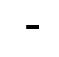

$-$
Negative electrode

128

12.5

1

100

0.471

0.357

1.5

26390

2000

0.05

0.53

$3.9 \times 10^{-14}$

$7.5 \times 10^{-11}$

0.363

$1.94 \times 10^{-11}$

0.5

0.0
Separator

76

$\begin{array}{ll}- & 8.5\end{array}$

1

3.8

0.297

0.444

0.724

1.5

22860

2000

2000

0.78

0.17

$1.0 \times 10^{-13}$

$7.5 \times 10^{-11}$

$7.5 \times 10^{-11}$

0.363

0.363

$2.16 \times 10^{-11}$

0.5

We compute $\sigma^{\mathrm{eff}}=\sigma \varepsilon_{s}, \kappa^{\mathrm{eff}}=\kappa \varepsilon_{e}^{\mathrm{brug}}, D_{e}^{\mathrm{eff}}=D_{e} \varepsilon_{e}^{\mathrm{brug}}$.

In the electrolyte, conductivity is a function of concentration:

$$
\begin{gathered}
\kappa\left(c_{e}\right)=4.1253 \times 10^{-2}+5.007 \times 10^{-4} c_{e}-4.7212 \times 10^{-7} c_{e}^{2} \\
+1.5094 \times 10^{-10} c_{e}^{3}-1.6018 \times 10^{-14} c_{e}^{4} .
\end{gathered}
$$

For the negative electrode, the open-circuit potential function is:

$$
U_{\mathrm{ocp}}(\theta)=-0.16+1.32 \exp (-3.0 \theta)+10.0 \exp (-2000.0 \theta)
$$

For the positive electrode, the open-circuit potential function is:

$$
\begin{aligned}
& U_{\text {ocp }}(\theta)=4.19829+0.0565661 \tanh (-14.5546 \theta+8.60942) \\
&-0.0275479\left[\frac{1}{(0.998432-\theta)^{0.4924656}}-1.90111\right] \\
&-0.157123 \exp \left(-0.04738 \theta^{6}\right)+0.810239 \exp [-40(\theta-0.133875)] .
\end{aligned}
$$

